\title{
Adaptive Reconnection-based Arbitrary Lagrangian Eulerian Method
}

\author{
Wurigen $\mathrm{Bo}^{\mathrm{c}}$, Mikhail Shashkov ${ }^{\mathrm{d}, *}$ \\ c Computational and Computer Science Division, CCS-2 \\ Los Alamos National Laboratory, Los Alamos, NM 87545, USA \\ ${ }^{d} \mathrm{X}$-Computational Physics, $\mathrm{XCP}-4$ \\ Los Alamos National Laboratory, Los Alamos, NM 87545, USA
}

\begin{abstract}
We present a new adaptive Arbitrary Lagrangian Eulerian (ALE) method. This method is based on the reconnection-based ALE (ReALE) methodology of references $[35,34,6]$. The main elements in a standard ReALE method are: an explicit Lagrangian phase on an arbitrary polygonal (in 2D) mesh in which the solution and positions of grid nodes are updated; a rezoning phase in which a new grid is defined by changing the connectivity (using Voronoi tessellation) but not the number of cells; and a remapping phase in which the Lagrangian solution is transferred onto the new grid. In the standard ReALE method, the rezoned mesh is smoothed by using one or several steps toward centroidal Voronoi tessellation, but it is not adapted to the solution in any way.

In the current paper we present a new adaptive ReALE method, A-ReALE, that is based on the following design principles. First, a monitor function (or error indicator) based on the Hessian of some flow parameter(s) is utilized. Second, an equi-distribution principle for the monitor function is used as a criterion for adapting the mesh. Third, a centroidal Voronoi tessellation is used to adapt the mesh. Fourth, we scale the monitor function to avoid very small and large cells and then smooth it to permit the use of theoretical results related to weighted centroidal Voronoi tessellation.

In the A-ReALE method, both number of cells and their locations are allowed to change at the rezone stage on each time step. The number of generators at each time step is chosen to guarantee the required spatial resolution in regions where monitor function reaches its maximum value.

We present all details required for implementation of new adaptive A-ReALE method and demonstrate its performance in comparison with standard ReALE method on series of numerical examples.
\end{abstract}

Keywords: Reconnection-based Arbitrary Lagrangian Methods (ReALE), R-Adaptation, H-Adaptation

\footnotetext{
* Corresponding author

Email address: shashkov@lanl.gov (Mikhail Shashkov)
} 


\section{Background and rationale}

The concept of a reconnection-based arbitrary Lagrangian-Eulerian method (ReALE) was introduced in [35]. As in most standard arbitrary LagrangianEulerian (ALE) methods, [23], the main elements in ReALE simulation are: an explicit Lagrangian phase in which the solution and grid are updated (without changing its connectivity), a rezoning phase in which a new grid is defined, and a remapping phase in which the Lagrangian solution is transferred onto the new rezoned grid.

The ReALE method described in [35] differs from the standard ALE method only in one element - it allows a connectivity change at the rezone stage. The rezone phase of ReALE includes both mesh movement and reconnection, which is done by using the machinery of Voronoi diagrams, [2]. That is, the ReALE rezone strategy consists of a special movement of the generators. The movement is close to Lagrangian in some sense, but also includes some smoothing based on the notion of centroidal Voronoi diagrams [11]. By construction, the Voronoi mesh is a valid mesh, and therefore each Lagrangian step starts with valid mesh. The main objective of [35] was to develop a robust reconnectionbased ALE method in which the averaged cell movement is close to Lagrangian. The ReALE method allows a complex simulation to be run to completion without user intervention while still achieving good accuracy. Examples of ReALE simulations can be found in $[35,34,6,20]$.

The ReALE methods have significant potential with respect to adaptivity. First of all, positioning (R-adaptivity) of generators at the rezone stage can be related to some error indicator. Secondly, the number of generators (which defines the number of cells) can change with time to refine the mesh where it is needed (H-adaptivity). However, in [35] there was no attempt to explore adaptivity within the framework of ReALE methods. In [5] we have explored R-adaptivity in ReALE framework, in which the number of cells is does not change, but the mesh is adapted to some monitor function.

In this paper we describe a new adaptive ReALE method (A-ReALE), in which both $\mathrm{R}$ - and $\mathrm{H}$-adaptivity is used. That is, number of generators that define the mesh at the rezone stage can increase and decrease as well as their locations.

The need for adaptive methods is well recognized, and there are numerous papers related to adaptation. See for example Chapter 14 in [33] and [24] and the corresponding references therein. For this reason we will not repeat here the standard arguments justifying the need for adaptive meshes.

According to [33], any adaptive method is composed of three main ingredients: an error estimator or indicator, an optimal-mesh criterion, and an algorithm for the mesh improvement strategy. These ingredients answer to the following questions: Where are the mesh changes required? How should the optimal mesh be defined? And how should the improved mesh be constructed? 
Our adaptive ReALE method is based on following well known basic design principles. ${ }^{1}$

The first design principle uses a monitor (error indicator) function based on the Hessian of some flow parameter(s). In general, the monitor function $\phi(\mathbf{x}, t)>0$ is a measure or indicator of the error [24, 33]. In an ideal case, its construction is based on error estimates. However, in reality, especially for non-linear hyperbolic problems, practitioners use much simpler and readily computable indicators of error.

The monitor function $\phi(\mathbf{x}, t)>0$ is known for any $\mathbf{x}$ only at initial time $t=0$. In general, it is given by its values on some mesh at a particular time moment $t^{n}$. We will denote these values by $\phi_{i}^{n}$, where $i$ index related to the some mesh entity and $n$ index identifies the time moment. To be used in the adaptation process, the monitor function has to satisfy some requirements. We will formulate these requirements after we describe how the monitor function is used to construct the adaptive mesh.

Second design principle uses an equi-distribution principle for the monitor function as a criterion for the definition of the adaptive mesh, [24, 33]. If we are given a polygonal computational domain $\Omega$ and the monitor function $\phi_{i}^{n}$, then the mesh, consisting of cells $\Omega_{i}$ such that $\cup_{i} \Omega_{i}=\Omega$, satisfies the equidistribution principle if

$$
\phi_{i}^{n}\left|\Omega_{i}\right|=E=\text { const } .
$$

We will call $E$ the equi-distribution level. If number of cells $N$ is given, then the equi-distribution level $E^{n}$ at time $t^{n}$ that can be achieved by equi-distribution, is

$$
E^{n}=\left(\sum_{i=1}^{N} \phi_{i}^{n}\left|\Omega_{i}\right|\right) / N
$$

As we will explain later, for the monitor function we are using

$$
\sum_{i=1}^{N} \phi_{i}^{n}\left|\Omega_{i}\right| \approx \int_{\Omega} \phi\left(\mathbf{x}, t^{n}\right) d V
$$

and therefore

$$
E^{n} \approx\left(\int_{\Omega} \phi\left(\mathbf{x}, t^{n}\right) d V\right) / N
$$

If we decide to achieve a particular equi-distribution level $E^{n}$ at time $t^{n}$, then it follows from (4) that we need the $N^{n}$ number of cells

$$
N^{n} \approx\left(\int_{\Omega} \phi\left(\mathbf{x}, t^{n}\right) d V\right) / E^{n} .
$$

\footnotetext{
${ }^{1}$ Let us note that to describe the main ideas we intentionally use a loose style of presentation to avoid lengthy definitions and explanations. In main text of the article we give strict definition of all notions and notations we use.
} 
Third design principle uses a centroidal Voronoi tessellation as a tool for creating an adaptive mesh that satisfies the equi-distribution principle [11]. To explain this, we need to recall some definitions $[2,11]$. Given a set of $N$ generators $\mathbf{G}=\left\{\mathbf{G}_{i}\right\}_{i=1}^{N}$, the Voronoi tessellation in an unbounded domain is defined as the spatial decomposition into cells $\Omega_{i}$

$$
\Omega_{i}=\left\{\mathbf{x} \in \mathbb{R}^{2}:\left\|\mathbf{x}-\mathbf{G}_{i}\right\| \leq\left\|\mathbf{x}-\mathbf{G}_{j}\right\|, \text { for } j=1, \ldots, N, j \neq i\right\},
$$

where $\|\cdot\|$ is the Euclidean distance. That is, the point $\mathbf{x}$ in $\Omega_{i}$ is closer to generator $\mathbf{G}_{i}$ than to any other generator from set $\mathbf{G}$. The region $\Omega_{i}$ is referred to as a Voronoi cell and the set $\left\{\Omega_{i}\right\}_{i=1}^{N}$ is referred to as a VT [9].

The center of mass of cell $\Omega_{i}$ with respect to weight function $\varphi(\mathbf{x})$ is

$$
c_{i}^{\varphi}=\frac{\int_{\Omega_{i}} \mathbf{x} \varphi(\mathbf{x}) d \mathbf{x}}{\int_{\Omega_{i}} \varphi(\mathbf{x}) d \mathbf{x}} .
$$

The Voronoi tessellation is called the centroidal Voronoi tessellation (CVT) with respect to weight $\varphi$ if $G_{i}=c_{i}^{\varphi}$, that is, if positions of the generators coincide with the center of mass of the corresponding Voronoi cell. The CVTs have several amazing and useful properties; in particular, in the asymptotic case of an infinite number of generators and a smooth weight function, cells of CVT are perfect hexagons in $2 \mathrm{D}$ and

$$
\sqrt{\varphi\left(\mathbf{G}_{i}\right)}\left|\Omega_{i}\right| \approx \sqrt{\varphi\left(\mathbf{G}_{j}\right)}\left|\Omega_{j}\right| .
$$

Therefore, if we set $\varphi=\phi^{2}$, we obtain a mesh that asymptotically satisfies the equi-distribution principle

$$
\phi\left(\mathbf{G}_{i}\right)\left|\Omega_{i}\right| \approx \phi\left(\mathbf{G}_{j}\right)\left|\Omega_{j}\right|, \quad \text { or } \quad \phi_{i}\left|\Omega_{i}\right| \approx \phi_{j}\left|\Omega_{j}\right| .
$$

All theoretical results about CVT have been proved assuming the weight function is infinitely smooth and number of generators goes to infinity. These conditions are sufficient for the corresponding theorems. In practice, to be used for the construction of an adaptive mesh, we need to guarantee some level of smoothness of the weight function and use a reasonably large number of generators.

The fourth design principle is the modification of the monitor function. This includes several steps. First step is smoothing. As already mentioned, the monitor function has to be sufficiently smooth to use CVT for the construction of a mesh that satisfies the equi-distribution principle. To construct a smooth monitor, we use ideas presented in the seminal paper [10]. The second step is scaling. As it is seen from equation (1), if the monitor is too large, then the corresponding cell will be very small. This can lead to a very small time step, because we use explicit time integration and time step has to satisfy CFL stability condition. If the monitor is very small, then the corresponding cell can be very large, and this can lead to loss of accuracy. Therefore, we would like to scale the smoothed monitor function to avoid very small and very large cells. 
We introduce "shape preserving scaling" such that the scaled monitor function preserves the "shape" of the smoothed monitor.

To describe our adaptive ReALE method (A-ReALE), we need to introduce an additional design principle, that is, how to define $E^{n}$ in (5) to determine the number of generators on each time step.

We consider two strategies. The first strategy is to keep the equi-distribution level $E^{n}$ constant in time, $E^{n}=E$. The second strategy is to maintain a prescribed, constant in time, spatial resolution for regions where the monitor has reached its maximum value.

In [5] we presented a general discussion related to the definition of the "efficiency" of adaptive methods and gave references to relevant papers. We will

not repeat this discussion here. The conclusion of this discussion is that it is very hard to define "efficiency" because it depends on the problem, computer architecture, goal of adaptation, and so on. Therefore, our opinion is that one must consider the new method as a new tool and present some performance characteristics of method in different situations. This will allow the potential user to make their own conclusions as to how useful the tool can be to their application. In this paper we follow this path.

The remainder of the paper is organized as follows. In Section 2, we present definitions related to Voronoi and weighted centroidal Voronoi meshes, consider their properties, and describe a related optimization problem that is the basis for construction of Voronoi meshes. In the same section we describe a new quadtree based initialization procedure for the optimization problem. In Section 3, we present the definition of the monitor function and describe smoothing and scaling procedures. In Section 4, we describe two adaptive ReALE strategies. A family of ReALE methods is described in Section 5. We describe standard nonadaptive ReALE methods as well as two new adaptive methods. In Section 6, we describe possible ways to compare the performance of standard non-adaptive adaptive ReALE methods. In Section 7, we present numerical examples that demonstrate different aspects of the new adaptive methods. The conclusion and future work is presented in Section 8.

\section{Constrained centroidal Voronoi tessellation}

In this Section, we describe the main tool that we use to construct meshes in rezone stage of adaptive ReALE methods, constrained centroidal Voronoi tessellation.

In Section 2.1, we give the definition of Voronoi tessellation in general nonconvex, non simply connected (holes) computational domains. The reader who is interested in more information about Voronoi tessellation can refer to $[2,41,11,12,27,13,50,39]$. In Section 2.2, we define centroidal Voronoi tessellation (CVT) with respect to some weight function and explain how the properties of such a tessellation can be used to construct a mesh that satisfies the equi-distribution principle. The computation of CVT, based on a variational formulation, is described in Section 2.4. It includes the description of 
a new initialization procedure based on a quad-tree approach as well as other implementation details.

\subsection{Voronoi Tessellation in a general domain}

Traditional Voronoi tessellation (VT) in an unbounded domain is described in the Background and rational Section for Voronoi cells corresponding to set of generators $\mathbf{G}=\left\{\mathbf{G}_{i}\right\}_{i=1}^{N}$ defined by (6).

If a $\mathrm{VT}$ is used in the discretization of a partial differential equation (PDE) in a bounded domain $\Omega$, then the boundaries of the cells of the VT must conform to the boundary of $\Omega$ so that the boundary condition of the PDE can be enforced.

We assume that computational domain $\Omega$ is a general non-convex nonsimply-connected domain. The external boundary of $\Omega$ is a closed polyline, that is, it consists of a ordered set of segments of straight lines, such that beginning of the one segment coincides with the end of another segment. We assume that segments of the external boundary are ordered in counter-clock-wise order, such that the computational domain is on the left side of the boundary. The internal boundaries are also closed polylines, but their segments are ordered clockwise with the computational domain again on the left of the internal boundaries. In simple terms, the computational domain is a polygon with polygonal holes. To keep integrity of the computational domain and represent the boundaries, we locate generators at the vertices of the polylines and refer to them as boundary generators. The generators that are inside the computational domain will be called internal generators. The set of internal generators are denoted by $\mathbf{G}_{I}=\left\{\mathbf{G}_{i}\right\}_{i=1}^{M}$; the set of boundary generators are denoted by $\mathbf{G}_{B}=\left\{\mathbf{G}_{i}\right\}_{i=M+1}^{N}$; and the full set of generators by $\mathbf{G}=\mathbf{G}_{I} \cup \mathbf{G}_{B}$.

The VT on $\Omega$ can be defined as (see, for example, [44, 45, 50, 47]):

$$
\Omega_{i}=\left\{\mathbf{x} \in \Omega \mid d\left(\mathbf{x}, \mathbf{G}_{i}\right) \leq d\left(\mathbf{x}, \mathbf{G}_{j}\right), \text { for } j=1, \ldots, N, j \neq i\right\} .
$$

where $d(\mathbf{x}, \mathbf{y})$ is the distance that incorporates visibility constraints

$$
d(\mathbf{x}, \mathbf{y})= \begin{cases}\|\mathbf{x}-\mathbf{y}\| & \text { if } \mathbf{x} \text { "sees" } \mathbf{y} \\ +\infty & \text { otherwise. }\end{cases}
$$

In this definition, $\mathbf{x}$ "sees" $\mathbf{y}$ when no line segment on $\partial \Omega$ intersects the line segment connecting $\mathbf{x}$ and $\mathbf{y}$, [41] In Fig.1, we illustrate VT for the flow over a cavity that will be described in Section 7 . The computational domain is nonconvex. To represent the boundary, we locate generators in the corners of the domain. One can see that cells corresponding to some boundary generators are non-convex. In Fig.2 we illustrate VT for a domain with circular holes. To represent the external boundary of the domain, we need to put four generators in the corners, and to represent a circular hole we need to put several generators on the circular boundary. The cells which correspond to boundary generators for internal boundary are slightly non-convex.

Let us note that Voronoi cells which correspond to internal generators are always convex, and Voronoi cells which correspond to boundary generators can be non-convex, but each cell always consists of only one piece. 


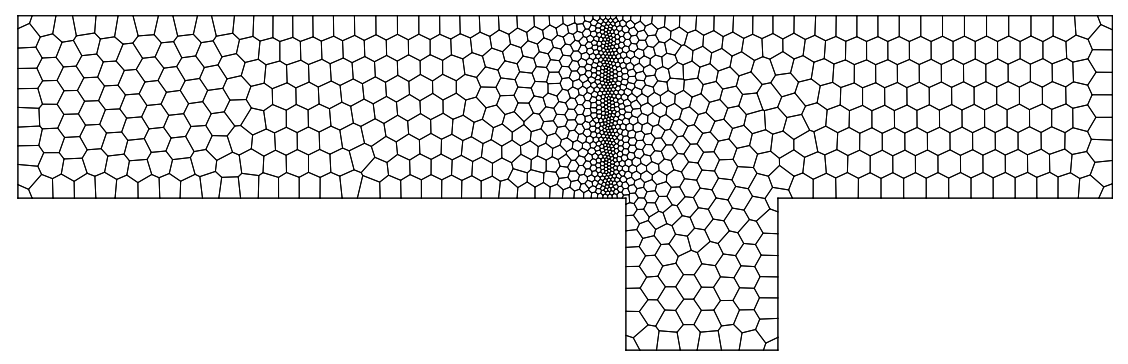

Figure 1: The VT of a non-convex domain - flow over cavity problem from Section 7 . To not make picture to busy we do not show generators.

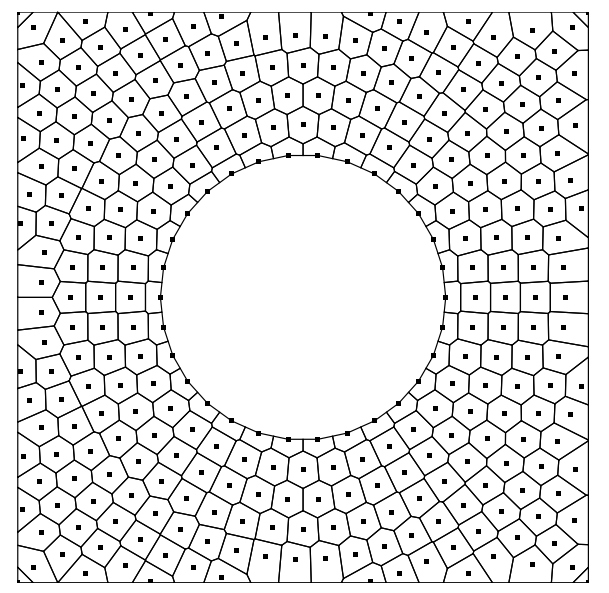

Figure 2: The VT for domain with circular hole. 


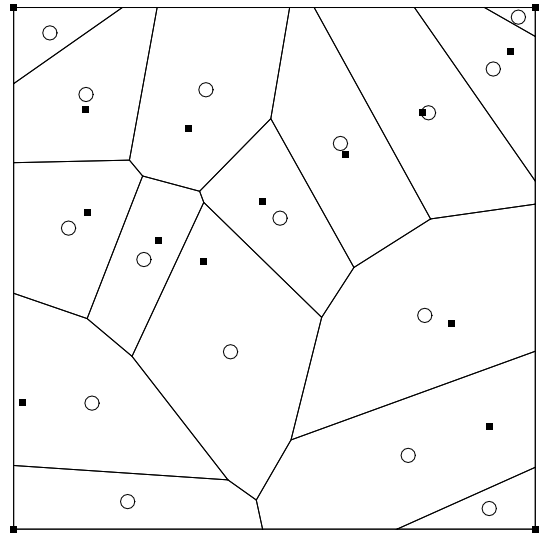

(a)

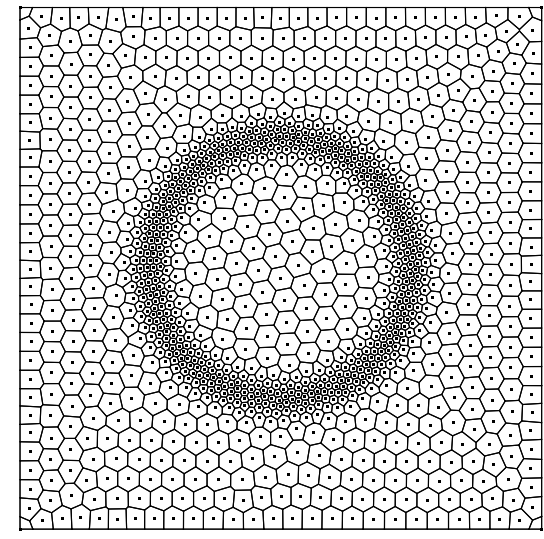

(b)

Figure 3: (a) VT for four generators on the boundary and 12 randomly placed generators. The generators marked by solid circles and centroids of corresponding cells marked bigger empty circles - clearly positions of the generators do not coincide with the positions of corresponding centroids. (b) CCVT with 1200 generators, which correspond to Gaussian weight function $\varphi(r)=e^{-\frac{(r-1 / 2)^{2}}{0.1}}$ with respect to radius $r=\sqrt{x^{2}+y^{2}}$.

\subsection{Constrained centroidal Voronoi tessellation}

Consider a weight function $\varphi(\mathbf{x})>0$ defined on $\Omega$. For each Voronoi cell $\Omega_{i}$, we can define its mass centroid $\mathbf{G}_{i}^{*}$ by

$$
\mathbf{G}_{i}^{*}=\frac{\int_{\Omega_{i}} \mathbf{x} \varphi(\mathbf{x}) d \mathbf{x}}{\int_{\Omega_{i}} \varphi(\mathbf{x}) d \mathbf{x}}, i=1, \ldots, N
$$

We refer to a VT as a constrained centroidal Voronoi tessellation (CCVT) if and only if

$$
\mathbf{G}_{i}=\mathbf{G}_{i}^{*}, i=1, \ldots, M .
$$

In this definition, the generators on the boundary are not subject to (10). The boundary generators are fixed to maintain the integrity of the domain shape, which is the simplest case of CCVTs in [39].

In left panel of Figure 3, we present a VT for four generators on the boundary and 12 randomly placed generators. The generators marked by solid circles and centroids of corresponding cells marked by bigger empty circles. Clearly, positions of the generators do not coincide with the positions of the corresponding centroids. In right panel of Figure 3, we present the CCVT (1200 generators) for a weight function which is defined as follows

$$
\varphi(r)=e^{-\frac{(r-1 / 2)^{2}}{0.1}}, r=\sqrt{x^{2}+y^{2}} .
$$

Given any set of generators $\left\{\mathbf{x}_{i}\right\}_{i=1}^{N}$ on $\bar{\Omega}$ and any tessellation $\left\{V_{i}\right\}_{i=1}^{N}$, we 
define the corresponding energy functional by

$$
\mathcal{F}\left(\left\{V_{i}\right\}_{i=1}^{N},\left\{\mathbf{x}_{i}\right\}_{i=1}^{N}\right)=\sum_{i=1}^{N} \int_{V_{i}} \varphi(\mathbf{x})\left\|\mathbf{x}-\mathbf{x}_{i}\right\|^{2} d \mathbf{x},
$$

In [39], it is shown that CCVTs are solutions of the problem

$$
\begin{gathered}
\min _{\left\{V_{i}\right\}_{i=1}^{N},\left\{\mathbf{x}_{i}\right\}_{i=1}^{N}} \mathcal{F}\left(\left\{V_{i}\right\}_{i=1}^{N},\left\{\mathbf{x}_{i}\right\}_{i=1}^{N}\right), \\
\text { subject to } \mathbf{x}_{j}=\mathbf{G}_{j}, j=M+1, \ldots, N,
\end{gathered}
$$

that is, boundary generators are fixed. If a CCVT with its generators is a global minimum of $\mathcal{F}$, it is called an optimal CCVT.

For an optimal CCVT with a smooth weight function, the internal Voronoi cells have many useful geometric properties as the number of generators gets sufficiently large [17]:

- Gersho's conjecture: In the limit of an infinite number of generators, the internal Voronoi cells are congruent to a basic cell that depends on the dimension. The basic cell in 2D is the regular hexagon. Although Gersho's conjecture has been proved in 2D [19], it remains open for three and higher dimensions.

- Based on Gersho's conjecture, the following relation holds for the internal Voronoi cells:

$$
\varphi\left(\mathbf{G}_{i}^{*}\right) h_{i}^{d+2} \approx \varphi\left(\mathbf{G}_{j}^{*}\right) h_{j}^{d+2},
$$

where $h_{i}$ is the diameter of the Voronoi cell $i$

$$
h_{i}=2 \max _{\mathbf{y} \in \Omega_{i}}\left\|\mathbf{y}-\mathbf{G}_{i}\right\|
$$

and $d$ is the dimension of space. Since all the internal Voronoi cells are asymptotically congruent to a basic cell, (14) can be rewritten in terms of the cell areas

$$
\varphi\left(\mathbf{G}_{i}^{*}\right)\left|\Omega_{i}\right|^{1+2 / d} \approx \varphi\left(\mathbf{G}_{j}^{*}\right)\left|\Omega_{j}\right|^{1+2 / d},
$$

where $\left|\Omega_{i}\right|$ is the length/area/volume in $1 \mathrm{D} / 2 \mathrm{D} / 3 \mathrm{D}$ of the Voronoi cell $i$.

\subsection{CCVT and construction of a mesh satisfying equi-distribution}

The properties of CCVT summarized at the end of the previous Section suggest that an optimal CCVT can be used to construct polygonal meshes that satisfy the equi-distribution condition. If we define a weight function $\varphi=\phi^{2}$ for a monitor function $\phi$ on a $2 \mathrm{D}$ domain, then using (16), on the optimal CCVT we have

$$
\phi\left(\mathbf{G}_{i}^{*}\right)\left|\Omega_{i}\right| \approx \phi\left(\mathbf{G}_{j}^{*}\right)\left|\Omega_{j}\right| .
$$

Therefore, the equi-distribution condition with respect to $\phi$ is satisfied asymptotically. The right panel of Figure 3 demonstrates this property. 
If number of generators is given, then according to (2) we will be able to achieve the following equi-distribution level on CCVT mesh with weight function $\varphi=\phi^{2}$

$$
E \approx\left(\int_{\Omega} \phi d V\right) / N
$$

If the desired level of the equi-distribution $E$ is given, then the total number of generators needed to achieve it is defined by equation (18)

$$
N_{\Omega} \approx\left(\int_{\Omega} \phi d V\right) / E
$$

Equation (19) is true for any domain and therefore the knowledge of $E$ allows us to estimate the number of generators needed to achieve this level of the equi-distribution in any subdomain $\omega$ of $\Omega$ as follows

$$
N_{\omega} \approx\left(\int_{\omega} \phi d V\right) / E
$$

This observation will be used as basis for the initialization procedure for computation of CCVT described in Section 2.4.2.

\subsection{Computation of CCVTs}

\subsubsection{Minimization of the energy functional}

CCVTs defined in (10) can be computed in the same way as CVTs. In this paper, we use a quasi-Newton method, the limited memory Broyden-FletcherGoldfarb-Shanno algorithm (L-BFGS) for CCVT computation [31]. To use LBFGS for CCVT computation, we need to define the objective function to be minimized. We use the following energy functional in L-BFGS that has the same minimizer as (13)

$$
\begin{aligned}
& \mathcal{K}\left(\left\{\mathbf{x}_{i}\right\}_{i=1}^{N}\right)=\sum_{i=1}^{N} \int_{\Omega_{i}} \varphi(\mathbf{x})\left\|\mathbf{x}-\mathbf{x}_{i}\right\|^{2} d \mathbf{x}, \\
& \min _{\left\{\mathbf{x}_{i}\right\}_{i=1}^{N}} \mathcal{K}\left(\left\{\mathbf{x}_{i}\right\}_{i=1}^{N}\right), \text { subject to } \mathbf{x}_{j}=\mathbf{G}_{j}, j=M+1, \ldots, N .
\end{aligned}
$$

where $\Omega_{i}$ are the Voronoi cells corresponding to the $\mathbf{x}_{i}$. Since the independent variables in (21) are the coordinates of the internal generators, we rewrite (12) as a new energy functional

$$
\mathcal{E}\left(\left\{\mathbf{x}_{i}\right\}_{i=1}^{M}\right)=\mathcal{K}\left(\left\{\mathbf{x}_{i}\right\}_{i=1}^{N}\right),
$$

L-BFGS also needs the gradient of $\mathcal{E}$, which is given in [11]:

$$
\frac{\partial \mathcal{E}}{\partial \mathbf{x}_{i}}=2\left(\mathbf{x}_{i}-\mathbf{G}_{i}^{*}\right) \int_{\Omega_{i}} \varphi(\mathbf{x}) d \mathbf{x}
$$


where $\mathbf{G}_{i}^{*}$ is the mass centroid of $\Omega_{i}$. The stopping criterion of L-BFGS is provided as follows. We define an averaged deviation

$$
D_{\text {ave }}=\frac{1}{M} \sum_{i=1}^{M} D_{i},
$$

where $D_{i}=\left\|\mathbf{x}_{i}-\mathbf{G}_{i}^{*}\right\| / h_{i}$ and $h_{i}$ is defined in (15). It can be seen from (23) that $D_{\text {ave }}=0$ if and only if $\partial \mathcal{E} / \partial \mathbf{x}_{i}=0, i=1, \ldots, M$. Thus $D_{\text {ave }}$ can be used to check the convergence of the CCVT computation. In this paper, we use a stopping criterion $D_{\text {ave }}<5 \cdot 10^{-4}$.

\subsubsection{Initialization for CCVT computation}

The convergence of the minimization algorithm described in previous section strongly depends on the initial guess for the positions of the generators. There are two main known approaches to initializing the position of the generators. In first class of methods, the initial guess is provided by some metric based mesh generation software $[13,14,40]$. In second class of method, probabilistic algorithms are used, [36, 26, 43].

In this section, we propose a new quad-tree based initialization for CCVT computation, that is as efficient as the mesh generation based method and is much easier to implement. This new method is based on exploiting the equidistribution property of CCVT.

In the beginning of the rezone phase, we have the Lagrangian mesh $\mathcal{M}^{L}$, that is the result of previous time step. Let us denote cells of this mesh by $\Omega_{i}^{L}$, and the centroids of these cells by $\mathbf{G}_{i}^{L}$. Let us also denote the number of cells in $\mathcal{M}^{L}$ by $N^{L}$. On each cell $\Omega_{i}^{L}$ we can compute the monitor function $\phi$ and the integral of the monitor function

$$
\Phi_{i}=\int_{\Omega_{i}^{L}} \phi(\mathbf{x}) d \mathbf{x} .
$$

For a given equi-distribution level $E$, we can estimate total number of generators required to achieve this resolution by using equation (19), in which we use equation (25) to estimate the right hand side

$$
N_{\text {total }}^{r e q}=\left\lfloor\left(\sum_{i=1}^{N_{L}} \Phi_{i}\right) / E\right\rfloor,
$$

where $\lfloor\cdot\rfloor$ means "the largest integer not greater than".

The goal of the initialization procedure is to distribute $N_{\text {total }}^{r e q}$ generators in the domain $\Omega$ in such a way that density of their distribution is close to what is expected from a mesh satisfying the equi-distribution principle. This will be achieved by starting with set of centroids of the Lagrangian mesh $\left\{\mathbf{G}_{i}^{L}\right\}$ and the adding new generators to this set or deleting generators from the set of $\left\{\mathbf{G}_{i}^{L}\right\}$. It is very important to start the process with set $\left\{\mathbf{G}_{i}^{L}\right\}$ because the positions of 
the centroids of the Lagrangian mesh reflect features of the flow and we do not want to perturb them.

Let us now recall the basic definitions and some terminology of a quad-tree in 2D [16]. Let $B$ be the bounding box of the entire computational domain. We refer to $B$ as the root of the quad-tree. $B$ is subdivided into 4 equally-sized quadtree cells, each of which being recursively subdivided several times. The children obtained by subdividing the cell are identified by their relative position within the parent cell: $\{N W, N E, S W, S E\}$. If a stopping criterion is reached in a quad-tree cell, we stop subdividing the quad-tree cell and call it a terminal cell or a leaf.

Now we will describe the algorithm that controls the subdivision of a quadtree cell. Let us denote the generic quad-tree cell by $\sigma$. At the beginning of the process $\sigma=B$. Now if $\sigma$ is a generic cell of the quad-tree, we need to decide when to stop subdividing it, that is to declare it to be a terminal cell or leaf and what final action to take with respect to this terminal cell. This final action eventually suppose to bring number of generators in this terminal cell to number of required generators. It may happen that we need to add some generators in addition to existing centroids of the cells from $\mathcal{M}^{L}$ or we need to remove some centroids or do nothing. In our algorithm these decisions will be based on analysis of two numbers: the estimate for the number of generators required to achieve the desired equi-distribution level $E$ in cell of quadtree $\sigma$ $N_{\sigma}^{r e q}$; and number of actual centroids of cells from mesh $\mathcal{M}^{L}$ that are in $\sigma$ $N_{\sigma}^{L}$. Let us note that at first step, when $\sigma=B, N_{\sigma}^{L}=N^{L}$ and $N_{\sigma}^{r e q}=N_{\text {total }}^{r e q}$. Both of these numbers are nonzero.

Let us describe how these two numbers are computed in general. First we define $\tilde{\sigma}$ as an approximation for $\sigma$ as union of cells $\Omega_{i}^{L}$ of Lagrangian mesh $\mathcal{M}^{L}$ such that their centroids belong $\sigma$

$$
\tilde{\sigma}=\bigcup_{i: \mathbf{G}_{i}^{L} \in \sigma} \Omega_{i}^{L} .
$$

The number of cells that defines $\tilde{\sigma}$ will be denoted by $N_{\sigma}^{L}$. In figure 4 we graphically demonstrate the definition of $\tilde{\sigma}$ in general case. There is no ambiguity in definition of this number. If $N_{\sigma}^{L}=0$ then there is no simple way to estimate the number of required generators in $\sigma$ and we declare this cell terminal and do not place any generators in $\sigma$.

During the subdivision process, we want the total number of generators to be equal to $N_{\text {total }}^{r e q}$ and, because definition of number of needed generators involves division of rational numbers and "the largest integer not greater than" operation, we compute the number of needed generators in two steps during subdivision.

Let us assume that we know $N_{\sigma}^{r e q}$ (which we do at the very first step of quad-tree construction). Now suppose we decided to subdivide this cell $\sigma$ into four subcells $\sigma_{k}: k \in\{N W, N E, S W, S E\}$. Using the definition of $\tilde{\sigma}$ we can 


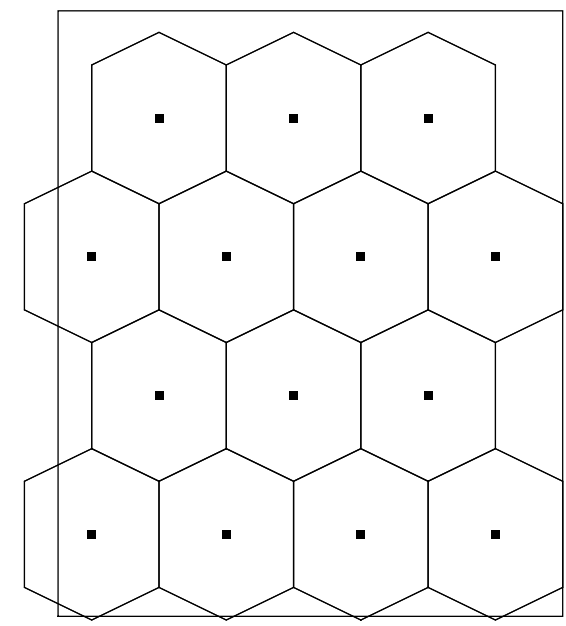

(a)

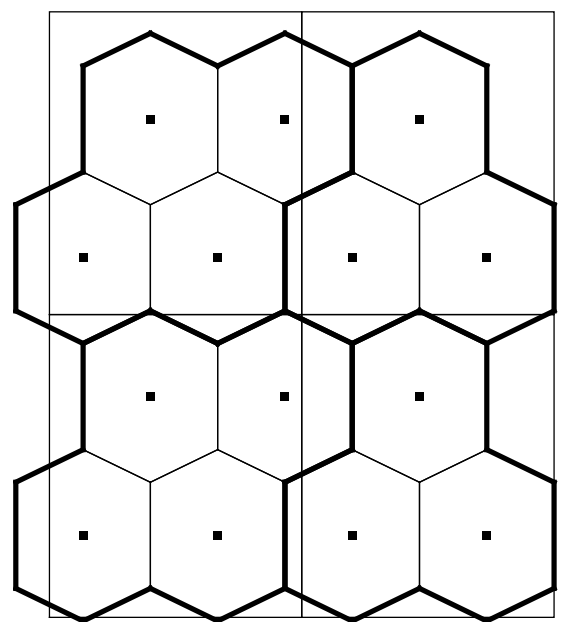

(b)

Figure 4: Quad-tree cells and its associated cells. The dots are the centroids of the cells. (a) a parent quad-tree cell. (b) The four children of the parent. The bold lines represent the boundaries of the domains associated with the children.

make a preliminary estimate of number of generators required for each of $\sigma_{k}$

$$
N_{\sigma_{k}}^{r e q}=\left\lfloor\left(\sum_{i: G_{i} \in \sigma_{k}} \Phi_{i}\right) / E\right\rfloor .
$$

It can happen that

$$
N_{\sigma}^{r e q} \neq \sum_{k} N_{\sigma_{k}}^{r e q}
$$

If we define

$$
p=N_{\sigma}^{r e q}-\sum_{k} N_{\sigma_{k}}^{r e q},
$$

then in general $0 \leq p \leq 4$. To keep total number of needed generators constant in the case when $p \neq 0$, we increase some or all $N_{\sigma_{k}}^{r e q}$ by one depending on value of $p$, such that after modification $N_{\sigma}^{r e q}=\sum_{k} N_{\sigma_{k}}^{r e q}$. If some of cells $\sigma_{k}$ have no $\mathbf{G}_{i}$ in it then, as we have mentioned before, it is declared terminal and does not participate in the described process of correction of the number of required generators.

Now for a generic quad-tree cell $\sigma$ we know two numbers $N_{\sigma}^{r e q} \geq 0$ and $N_{\sigma}^{L} \geq 1$. First decision we need to make is the quad-tree cell is terminal or not. We first check $N_{\sigma}^{L}$. If $N_{\sigma}^{L}=1$, we declare this cell terminal and need to put required number of generators $N_{\sigma}^{r e q}$ in this one Lagrangian cell. Because we already have one generator in $\sigma$, we need to add $N_{\sigma}^{r e q}-1$ additional generators. This can be done in different ways, and we have found that specific placement 
of additional generators does not make much difference. However, care has to be taken that all generators have distinct coordinates.

If $N_{\sigma}^{L} \geq 2$, we first check $N_{\sigma}^{r e q}$. If $N_{\sigma}^{r e q}=0$ or $N_{\sigma}^{r e q}=1$, we declare this cell terminal and, correspondingly, remove all generators or keep only one randomly chosen generator from $N_{\sigma}^{L}$ generators in cell $\sigma$. In all other possible cases we continue the subdivision process until all quad-tree cells declared terminal.

The above CCVT computation relies on the evaluation of the integrals in (9) and (23) on Voronoi cells. One approach to numerical evaluation is described in [5] and we will not repeat it here.

\subsubsection{Numerical examples - CCVT construction}

In this section we compare the performance of our new initialization algorithm with two other published initialization algorithms. In first algorithm the initial generators are placed on the domain with a Monte Carlo method [26]. The density function is given by $\varphi$. In second algorithm the initial generators are placed on the vertices of a Delaunay mesh generated from a metric based mesh generation software BAMG [22].

BAMG needs the required edge length as its input, which is computed as follows: Assume the required edge length is $h(\mathbf{x})$, we have $\left|\Omega_{i}\right| \sim h(\mathbf{x})^{2}$. From (16) we obtain

$$
h(\mathbf{x}) \sim \varphi(\mathbf{x})^{-1 / 4} .
$$

This relation has been used in Delaunay mesh optimization [14].

The above two initialization methods are hereby referred to as Random and BAMG. All the tests are run on a desktop computer with $2.33 \mathrm{GHz}$ Intel Xeon CPU and 8GB RAM. We compare the convergence speed and the computational time of L-BFGS using different initializations. In all methods to be compared, a CCVT function call consists of constructing the VT of the current generators and computing the energy functional and its gradient. A CCVT function call may be invoked multiple times in each iteration due to line search in L-BFGS.

To measure how much $E_{i}$ varies from the desired level $E$ (which is at the same time is average of the $E_{i}$ 's), we compute the standard deviation

$$
E_{\text {std }}=\sqrt{\frac{\left(E_{i}-E\right)^{2}}{M}} .
$$

We present comparison on two examples. The first example is for CCVT in convex square domain, and the second example in non-convex star-shaped domain. In both examples the monitor function is given analytically and we first compute values of the monitor at the vertices of the triangular background mesh (no modification of monitor is performed) and then apply algorithms for computing integrals and so on as described in [5].

Comparison - Convex Square Domain. The weight function is given by $\varphi(\mathbf{x})=\left(1+8 e^{-\|\mathbf{x}\|^{2} / 0.32}\right)^{2}$ on $[-1,1] \times[-1,1]$. We first compare the performance of the L-BFGS using three different initializations with 2856 generators. The quad-tree algorithm needs a mesh on which monitor is given $\left(\mathcal{M}^{\mathcal{L}}\right.$ mesh in context of ReALE method) - in this Section we will call it reference mesh. In 


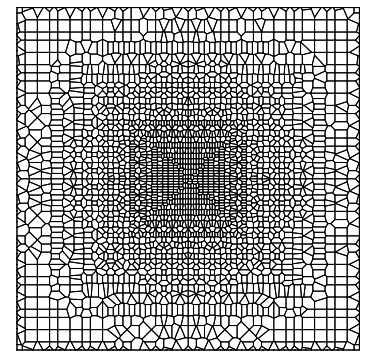

(a)

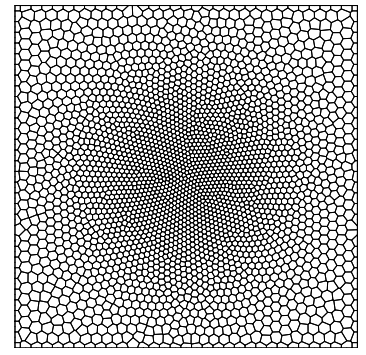

(a1)

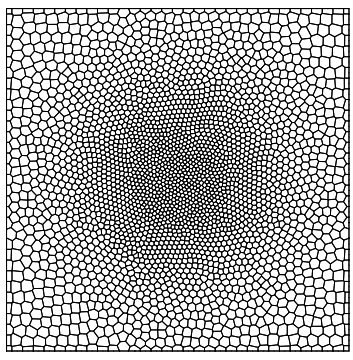

(b)

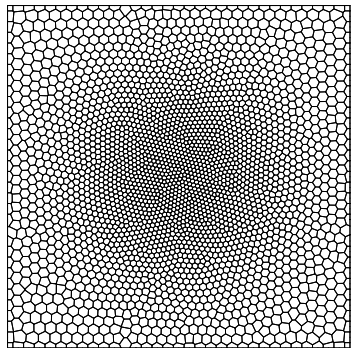

(b1)

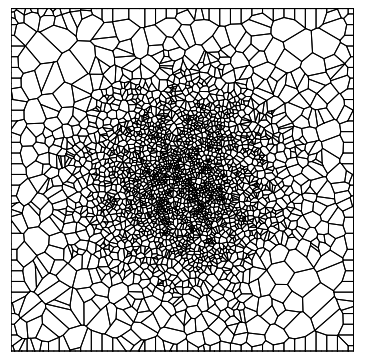

(c)

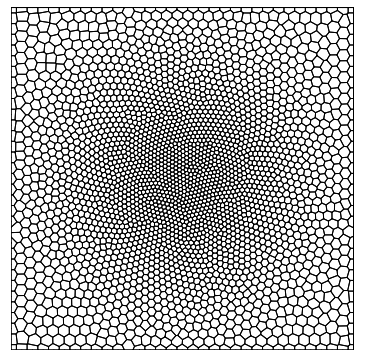

$(\mathrm{c} 1)$

Figure 5: CCVTs with 2856 generators using different initialization. (a)-(c): the initial VTs. (a1)-(c1): the results after L-BFGS. (a),(a1): Quad-tree. (b),(b1): BAMG. (c),(c1): Random.

this example we use a structured square mesh with $42 \times 42$ cells as reference mesh. The background mesh is triangular mesh obtained by connection of the generators with its neighbors in the reference mesh, the details are presented in [5].

Figure 5 (a1)-(c1) show the results computed with different initializations. It can be seen that the mesh quality after L-BFGS is similar although the initial meshes are quite different. Figure 6 shows that after L-BFGS, $E_{i}$ 's are distributed around the $E$ and three initializations give similar results.

Then, we compare the efficiency of the three initializations using 5798 and 11300 generators. The reference meshes are structured meshes with $58 \times 58$ and $84 \times 84$ cells. Table 1 shows both BAMG and Quad-tree have similar performance and are more efficient than Random. 


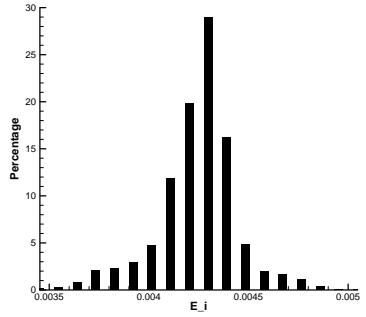

Quad-tree

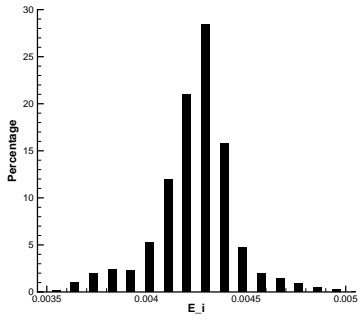

BAMG

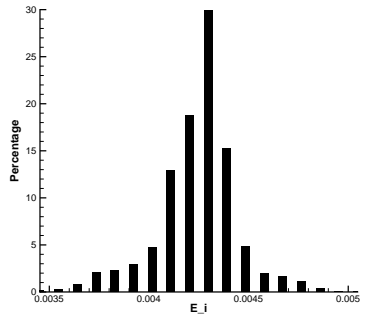

Random

Figure 6: The distribution of $E_{i}$ from CCVTs with 2856 generators after L-BFGS. The desired equi-distribution level $E=0.00423$.

\begin{tabular}{|c|c|c|c|c|c|c|}
\hline gens. & Initialization & iter. & fcs. & time $(\mathrm{s})$ & $\mathcal{E}$ & $E_{\text {std }}$ \\
\hline \hline \multirow{3}{*}{2856} & Random & 104 & 105 & 21.6 & $7.794 \mathrm{e}-3$ & $1.964 \mathrm{e}-4$ \\
\cline { 2 - 7 } & BAMG & 90 & 112 & 21.1 & $7.791 \mathrm{e}-3$ & $1.978 \mathrm{e}-4$ \\
\cline { 2 - 7 } & Quad-tree & 87 & 89 & 19.3 & $7.791 \mathrm{e}-3$ & $1.998 \mathrm{e}-4$ \\
\hline \hline \multirow{3}{*}{5798} & Random & 169 & 179 & 77.3 & $3.829 \mathrm{e}-3$ & $1.028 \mathrm{e}-4$ \\
\cline { 2 - 7 } & BAMG & 100 & 101 & 40.2 & $3.833 \mathrm{e}-3$ & $1.036 \mathrm{e}-4$ \\
\cline { 2 - 7 } & Quad-tree & 101 & 102 & 38.1 & $3.835 \mathrm{e}-3$ & $9.242 \mathrm{e}-5$ \\
\hline \hline \multirow{3}{*}{11300} & Random & 189 & 195 & 177.1 & $1.962 \mathrm{e}-3$ & $5.085 \mathrm{e}-5$ \\
\cline { 2 - 7 } & BAMG & 125 & 126 & 92.7 & $1.963 \mathrm{e}-3$ & $5.053 \mathrm{e}-5$ \\
\cline { 2 - 7 } & Quad-tree & 127 & 128 & 86.7 & $1.965 \mathrm{e}-3$ & $4.72 \mathrm{e}-5$ \\
\hline
\end{tabular}

Table 1: Comparison - Square Example: gens. is the number of generators; iter. is the number of iterations; fcs. is the number of CCVT functions calls. $\mathcal{E}$ and $E_{s t d}$ are defined in $(22)$ and $(31)$

The left panel of Figure 7 shows the history of the energy functional. Although the initial energy functional from Quad-tree is larger than that from BAMG, it decreases quickly during the first 30 L-BFGS iterations and the energy functionals of all initializations reach almost the same value after L-BFGS. Similar behavior can be seen for $D_{\text {ave }}$ and $E_{\text {std }}$ from the middle and the right panels of Figure 7.

Comparison - Non-convex Star-shaped Domain. The weight function is given by $\varphi(\mathbf{x})=\left(1+8 e^{-\|x\|^{2} / 0.02}\right)^{2}$ on the star-shaped domain. We compare the performance of L-BFGS with the three initializations using 2732 generators. The reference mesh is constructed as follows. We first take a structured mesh with $42 \times 42$ cells on the bounding box of the domain. Then we consider centers of these cells and if its belong to the star-shaped domain then we consider it as internal generator. The vertices of the star are boundary generators. Using these generators we construct VT, which will be considered as reference mesh. The background mesh is constructed from reference mesh in the standard way.

Figure 8 shows the VTs before and CCVT after L-BFGS. Figure 9 shows the distribution of $E_{i}$ 's after L-BFGS. We see the mesh quality is similar for the 

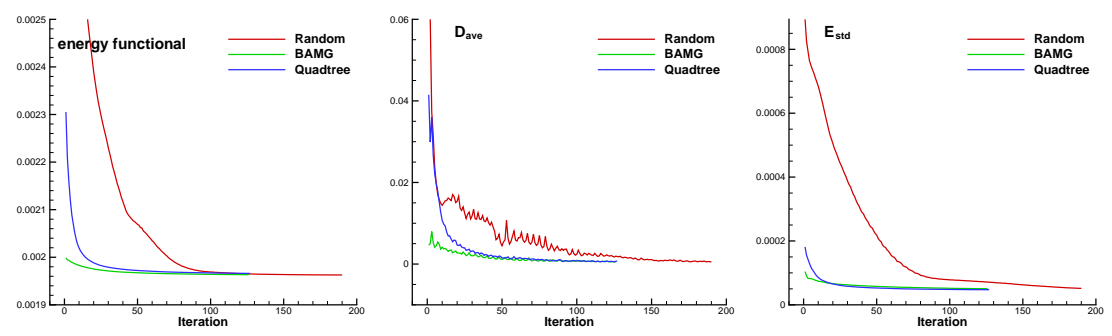

Figure 7: History of $\mathcal{E}, D_{a v e}$ and $E_{s t d}$ vs. number of iteration in L-BFGS iterations with 11300 generators.

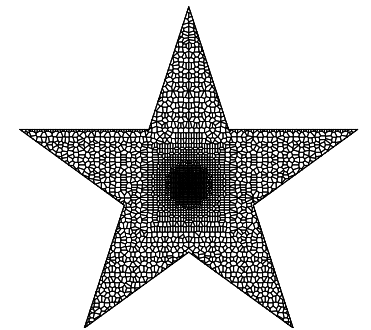

(a)

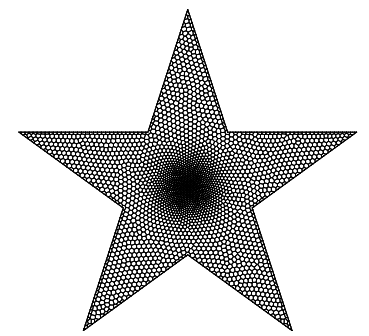

(a1)

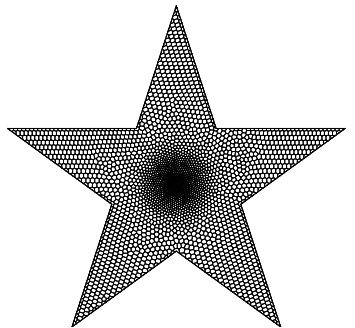

(b)

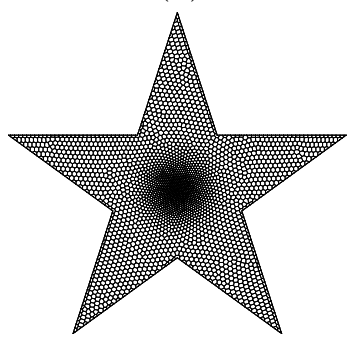

(b1)

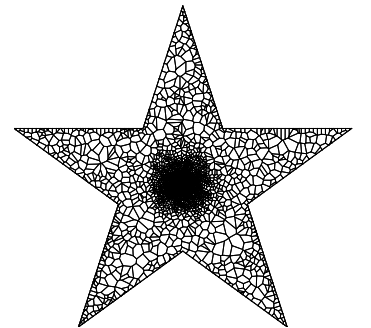

(c)

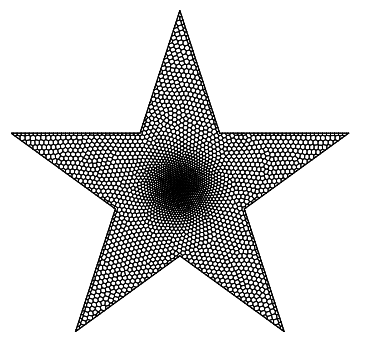

(c1)

Figure 8: CCVTs on a star-shape domain with 2732 generators using different initialization. (a)-(c): the initial VTs. (a1)-(c1): the CCVT results after L-BFGS. (a),(a1): Quad-tree. (b),(b1): BAMG. (c),(c1): Random.

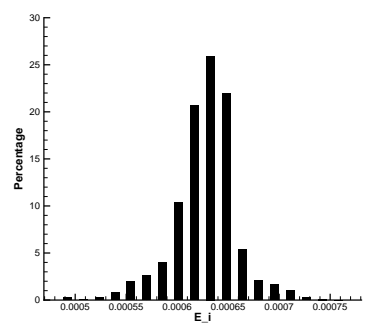

Quad-tree

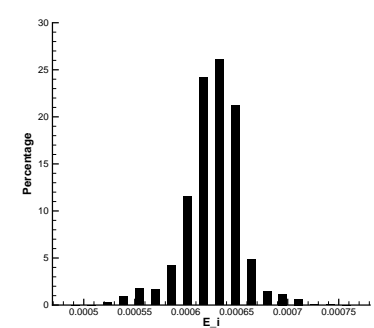

BAMG

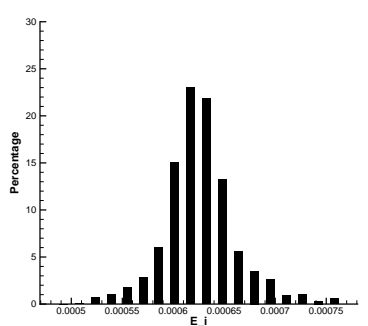

Random

Figure 9: The distribution of $E_{i}$ from CCVTs on a star-shape domain with 2732 generators after L-BFGS. The desired equi-distribution level $E=0.000626$. 

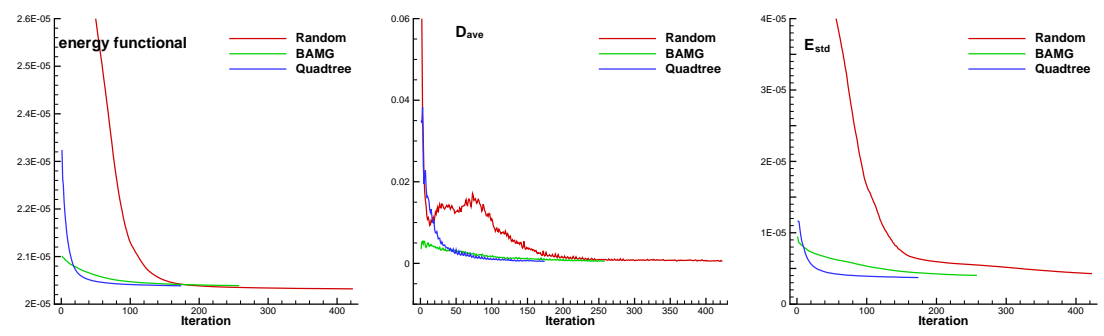

Figure 10: History of $\mathcal{E}, D_{\text {ave }}$ and $E_{\text {std }}$ vs. number of iterations in L-BFGS with 20489 generators on a star-shape domain.

different initializations. We also compare the efficiency of L-BFGS using 5229, 10624, 20489 generators. The reference meshes are constructed in similar way as before using $72 \times 72,103 \times 103$ and $143 \times 143$ square meshes in bounding box.

Table 2 shows the results of all the tested methods, in the same format as in the previous example. We also find that Quad-tree and BAMG has better performance compared to Random.

\begin{tabular}{|c|c|c|c|c|c|c|}
\hline gens. & Initialization & iter. & fcs. & time $(\mathrm{s})$ & $\mathcal{E}$ & $E_{\text {std }}$ \\
\hline \hline \multirow{3}{*}{5229} & Random & 292 & 417 & 100.4 & $7.946 \mathrm{e}-5$ & $1.944 \mathrm{e}-5$ \\
\cline { 2 - 7 } & BAMG & 183 & 187 & 55.7 & $8.001 \mathrm{e}-5$ & $1.745 \mathrm{e}-5$ \\
\cline { 2 - 7 } & Quad-tree & 154 & 156 & 38.8 & $7.986 \mathrm{e}-5$ & $1.579 \mathrm{e}-5$ \\
\hline \hline \multirow{3}{*}{10624} & Random & 346 & 352 & 217.9 & $3.941 \mathrm{e}-5$ & $8.636 \mathrm{e}-6$ \\
\cline { 2 - 7 } & BAMG & 234 & 237 & 144.5 & $3.943 \mathrm{e}-5$ & $6.870 \mathrm{e}-6$ \\
\cline { 2 - 7 } & Quad-tree & 178 & 179 & 101.2 & $3.949 \mathrm{e}-5$ & $7.282 \mathrm{e}-6$ \\
\hline \hline \multirow{3}{*}{20489} & Random & 422 & 458 & 571.2 & $2.032 \mathrm{e}-3$ & $4.280 \mathrm{e}-6$ \\
\cline { 2 - 7 } & BAMG & 227 & 231 & 304.1 & $2.039 \mathrm{e}-5$ & $4.026 \mathrm{e}-6$ \\
\cline { 2 - 7 } & Quad-tree & 173 & 174 & 196.8 & $2.038 \mathrm{e}-5$ & $3.724 \mathrm{e}-6$ \\
\hline
\end{tabular}

Table 2: Comparison - Non-convex star-shaped domain.

Figure 10 shows the history of $\mathcal{E}, D_{\text {ave }}$ and $E_{\text {std }}$. Both Quad-tree and BAMG converge faster than Random. Although the energy functional of Quad-tree is larger than that of BAMG, it converges faster in the first 10 iterations and reaches the final state with less iterations.

From the above examples, we see that the mesh quality after L-BFGS for all the initializations is similar. We also find both Quad-tree and BAMG give better initial guesses compared to Random. Although the initial guess from Quad-tree is not as good as that from BAMG, Quad-tree converges faster in the first few L-BFGS iterations and both reach the specified tolerance in a similar number of L-BFGS iterations. This leads to the similar performance for Quad-tree and BAMG in CCVT computation. 


\section{Monitor function}

In this section we describe how to compute monitor function values on background triangular mesh. The raw monitor function is first computed using eigenvalues of Hessian computed from flow parameter given on computational mesh. Next, we perform several modifications of the raw monitor. First, we smooth monitor to be able to use theoretical results related to CCVT, and then we scale the monitor to avoid too small or too big cells.

\subsection{Raw monitor}

We base the construction of the monitor function on an estimate of the interpolation error. Although it may not be directly related to the solution error, interpolation error provides important information on whether a mesh is suitable to approximate the solution of the PDE [7].

One of the options is to use a monitor function based on the L1 error norm of a linear interpolation [24]. This monitor is related to Hessian matrix. Let $H_{u}$ be the Hessian matrix of a scalar physical quantity $u$. $H_{u}$ is decomposed as

$$
H_{u}=\left(\begin{array}{cc}
\frac{\partial^{2} u}{\partial x^{2}} & \frac{\partial^{2} u}{\partial x \partial y} \\
\frac{\partial^{2} u}{\partial y \partial x} & \frac{\partial^{2} u}{\partial y^{2}}
\end{array}\right)=Q^{T}\left(\begin{array}{cc}
\lambda_{1} & 0 \\
0 & \lambda_{2}
\end{array}\right) Q,
$$

where $Q$ is the eigenvector matrix and the coefficients $\lambda_{i}$ are the eigenvalues of $H_{u}$. The monitor function based on the $\mathrm{L} 1$ norm error of a linear interpolation is $[24]$

$$
\phi=\max \left(\left|\lambda_{1}\right|,\left|\lambda_{2}\right|\right)^{1 / 2}=\sqrt{\left\|H_{u}\right\|},
$$

where $\left\|H_{u}\right\|$ is a spectral norm of the Hessian.

Knowing the nodal values of the physical state $u$, many methods can be used to compute the Hessian matrix components (second derivatives of $u$ ) at vertices. Here, we use a finite volume like approach [8]. The advantage of this approach is that it computes the Hessian matrix on a vertex by only using the nodal values of its adjacent vertices. Thus it is much faster than the classic use of least square approximation.

Let us present how the raw monitor based of Hessian looks like in 1D for exact solution of well-known Sod problem, [46] at $t=0.2$, projected to uniform mesh consisting of 200 cells - Fig. 11 (left panel). The raw monitor in this case is $\sqrt{\left|\partial^{2} u / \partial x^{2}\right|}$. It is approximated using simple finite difference formula. The raw monitor corresponding to Hessian is presented in the right panel in Fig.11.

\subsection{Monitor smoothing}

As we have already mentioned, the monitor function has to be sufficiently smooth to use CCVT for the construction of a mesh that satisfies the equidistribution principle. To construct a smooth monitor we use ideas presented in the seminal paper [10]. To obtain a smooth monitor function $\tilde{\phi}$ from the raw monitor function $\phi$, authors of [10] suggest solving

$$
\left(I-\alpha(1+\alpha) \delta^{2}\right) \tilde{\phi}=\phi
$$



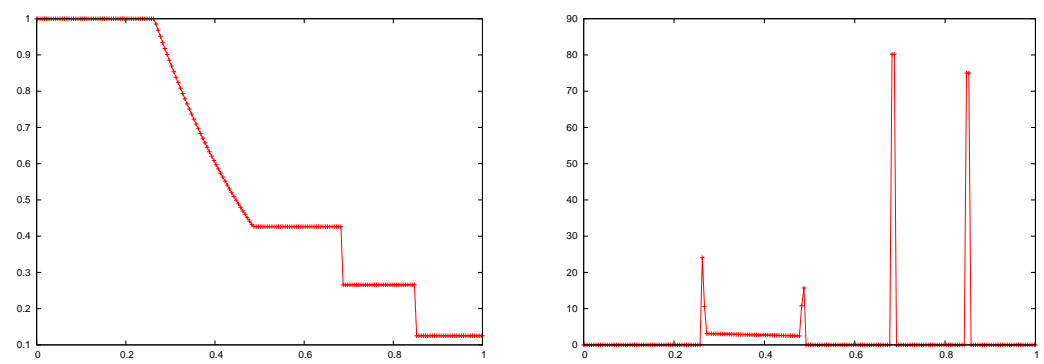

Figure 11: Sod problem: left panel - exact solution for the density profile at $t=0.2$ on the uniform mesh of 200 cells; right - raw monitor using Hessian.

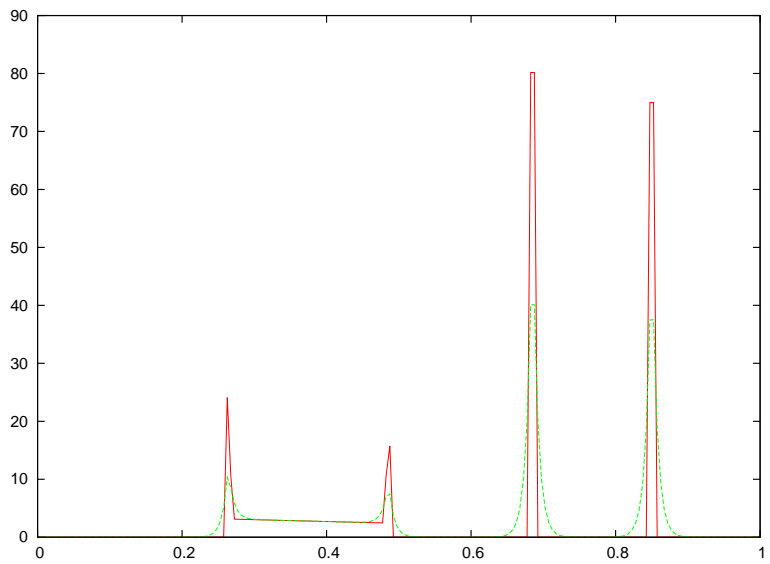

Figure 12: Sod problem: raw (red) and smoothed (green) monitors

for spatial smoothing, where $I$ is identity operator and $\delta^{2}$ is Laplacian in logical space. This equation is solved with zero Neumann boundary conditions. The smoothed monitor approximately satisfies the following conditions

$$
\alpha /(1+\alpha) \leq \tilde{\phi}_{i} / \tilde{\phi}_{j} \leq(1+\alpha) / \alpha,
$$

where $j$ is index of neighbor cell of the cell $i$. This process is "shape" preserving. And it also preserves total integral of the monitor function.

In Fig.12 we superimpose raw and smoothed monitors for the Sod solution for $\alpha=1$.

\subsection{Monitor scaling}

For our example, the raw monitor is zero in the regions where function is constant. In general, as it is seen from equation (1), if monitor is too big then the corresponding cell will be very small. This can lead to very small time step, 
because we use explicit time integration and the time step has to satisfy the CFL stability condition. If monitor is very small then the corresponding cell can be very big and this can lead to loss of accuracy. Therefore, we would like to scale the monitor function to avoid very small and very big cell. However, we would like to scale it in such way that modified monitor function preserves "shape" of the original monitor. We will call this process "shape preserving scaling" or just "scaling". We will denote scaled monitor by $\hat{\phi}$.

To construct $\hat{\phi}$, we first compute the average value of the smoothed monitor as follows

$$
\overline{\tilde{\phi}}=\sum_{i} \tilde{\phi}_{i}\left|\Omega_{i}\right| / \sum_{i}\left|\Omega_{i}\right| \approx \int_{\Omega} \phi d V /|\Omega| .
$$

If we use $\overline{\tilde{\phi}}$ as the monitor function, we will obtain uniform mesh consisting of hexagons in 2D (uniform mesh in 1D). Because

$$
\int_{\Omega} \overline{\tilde{\phi}} d V=\int_{\Omega} \phi d V
$$

Then to achieve the same equi-distribution level $E$ using $\bar{\phi}$ or $\phi$, we will need the same number of generators $N$. For a uniform mesh we have

$$
A_{i}=\bar{A}=|\Omega| / N
$$

and

$$
\bar{A} \bar{\phi}=E .
$$

For a mesh obtained from a smoothed monitor we have

$$
A_{i} \tilde{\phi}_{i}=E=A_{\min } \tilde{\phi}_{\max }=A_{\max } \tilde{\phi}_{\min }
$$

Therefore the variation of area size for a mesh corresponding to the smoothed monitor is

$$
A_{\min } / A_{\max }=\tilde{\phi}_{\min } / \tilde{\phi}_{\max }
$$

We construct the scaled monitor function as follows

$$
\hat{\phi}_{i}=\overline{\tilde{\phi}}+\beta\left(\tilde{\phi}_{i}-\overline{\tilde{\phi}}\right), \quad 0 \leq \beta \leq 1,
$$

such that $\beta=0$ will correspond to uniform mesh and $\beta=1$ corresponds to most "adaptive" mesh. The continuous analog of this definition is

$$
\hat{\phi}(\mathbf{x})=\overline{\tilde{\phi}}+\beta(\tilde{\phi}(\mathbf{x})-\overline{\tilde{\phi}}) \text {. }
$$

From equation (42) we can conclude that

$$
\nabla_{\mathbf{L}} \hat{\phi}=\beta \nabla_{\mathbf{L}} \tilde{\phi}, \quad \forall \mathbf{L}
$$

where $\nabla_{\mathbf{L}}$ is directional derivative in $\mathbf{L}$ direction. The equation (43) is definition of shape preservation. 


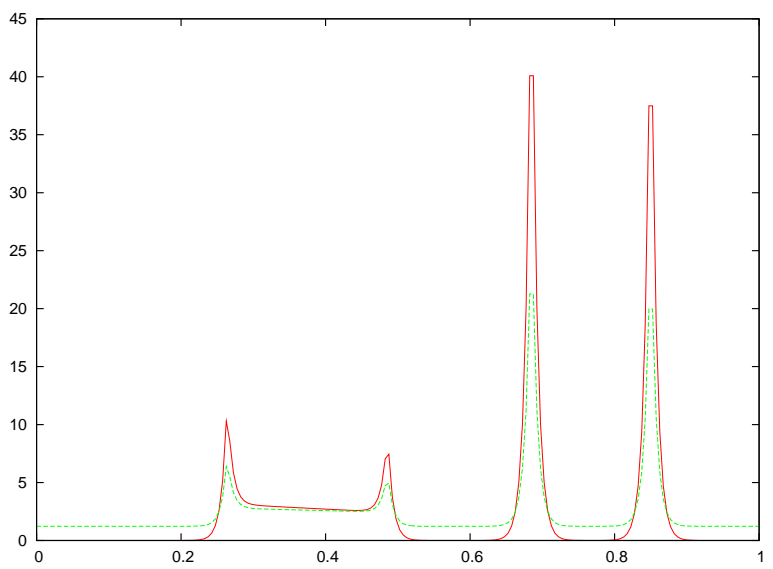

Figure 13: Sod problem: smoothed (red) and scaled (green) monitors

In Fig.13 we superimpose smoothed and scaled monitors for the Sod solution for $\beta=0.5$

One can see that the scaled monitor is "shape" preserving and has bigger minimum and smaller maximum in comparison with the smoothed monitor, and in particular its minimum is not zero.

Finally, in Fig.14 we plot the ratio of values of the monitor after smoothing and scaling in two neighboring cells as function of the coordinate. According to the inequality (34), the ratio of the value in the neighboring cells is between 2 and $1 / 2$ for $\alpha=1$.

\section{Adaptation strategies}

We consider two adaptation strategies. The first adaptation strategy, which leads to change of number of cells in time is based on keeping a fixed equidistribution level $E^{n}=E$ in time. Let us assume that $E$ is fixed in time, but the solution is changing in time (and therefore the monitor is changing). Then the number of generators $N^{n}$ needed to maintain this equi-distribution level at time $t^{n}$ is ${ }^{2}$

$$
N^{n} \approx\left(\int_{\Omega\left(t^{n}\right)} \phi\left(\mathbf{x}, t^{n}\right) d V\right) / E .
$$

We will call the adaption strategy based on equation (44) by A-ReALE(E=const). For the A-ReALE $(\mathrm{E}=$ const $)$ strategy $E$ is constant in time, and because the

\footnotetext{
${ }^{2}$ In the equation (44) we have dropped ${ }^{\wedge}$ in the notation of the monitor. In the rest of the paper, we will always assume that we are dealing with smoothed and scaled monitor.
} 


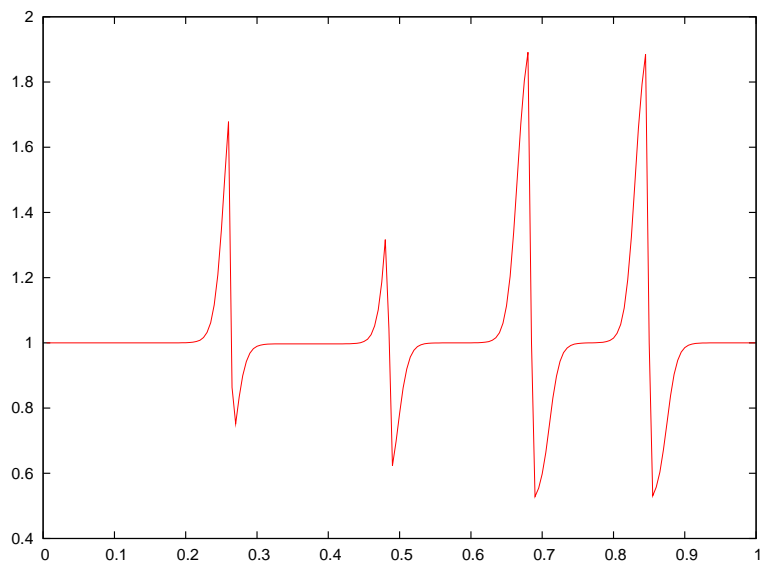

Figure 14: Sod problem: ratio of values of monitor after smoothing and scaling in two neighboring cells as function of the coordinate.

mesh satisfies the equi-distribution principle we have

$$
E=A_{\min }^{n} \phi_{\max }^{n} .
$$

Therefore, $A_{\min }$ and $A_{\max }$ are changing in time

$$
A_{\min }^{n}=E / \phi_{\max }^{n}, \quad A_{\max }^{n}=E / \phi_{\min }^{n} .
$$

The ratio of $A_{\min } / A_{\max }$ is also changing in time

$$
A_{\min }^{n} / A_{\max }^{n}=\phi_{\min }^{n} / \phi_{\max }^{n} .
$$

We also can change $E$ in time $\left(E^{n}\right)$, then we will have

$$
N^{n} \approx\left(\int_{\Omega\left(t^{n}\right)} \phi\left(\mathbf{x}, t^{n}\right) d V\right) / E^{n} .
$$

There are many possible strategies in defining $E^{n}$. In this paper we explore only one strategy which guarantees a prescribed level of spatial resolution in the regions where the monitor function reaches its maximum value. That is, we want mesh size, $h_{m i n}$, to be maintained for the spatial areas where the monitor function reaches its maximum. Because the cells of CVT are asymptotically perfect hexagons we can specify $A_{\min }=\min _{i}\left|\Omega_{i}\right| \sim h_{\min }^{2}$ for the required spatial resolution.

To explain this strategy we rewrite equation (1) as follows

$$
E^{n}=\phi_{i}^{n} A_{i}^{n},
$$

where, for sake of brevity, we use notation $A_{i}=\left|\Omega_{i}\right|$. The equation (49) is true for any cell and in particular for cells in which the monitor reaches its maximum 
value. For these cells, the area reaches its minimum value. Therefore, we can write

$$
E^{n}=\phi_{\max }^{n} A_{\min }^{n} .
$$

The monitor function is given and therefore, $\phi_{\max }^{n}$ is given. Consequently, if we want to maintain a spatial resolution fixed in time $A_{\min }^{n}=A_{\min }=$ const for regions where monitor reaches its maximum value, then we need to define the desired equi-distribution level depending on time as follows

$$
E^{n}=\phi_{\max }^{n} A_{\min } .
$$

Therefore using (51) and (48) we can conclude that to maintain spatial resolution $A_{\min }$ we need the following number of generators

$$
N^{n} \approx\left(\int_{\Omega\left(t^{n}\right)} \phi\left(\mathbf{x}, t^{n}\right) d V\right) /\left(\phi_{\max }^{n} A_{\min }\right) .
$$

We will call adaptation strategy based on $(52)$ by $\operatorname{A}-\operatorname{ReALE}\left(A_{\text {min }}=\text { const }\right)^{3}$.

Let us note that we do not have any control on $A_{\max }$, however, its value can be deduced from equation

$$
A_{\min } \phi_{\max }^{n}=A_{\max }^{n} \phi_{\min }^{n},
$$

which follows from the equi-distribution principle. This equation leads to following expression for $A_{\max }^{n}$

$$
A_{\max }^{n}=A_{\min } \frac{\phi_{\max }^{n}}{\phi_{\min }^{n}} .
$$

This means that $A_{\max }$ depends on time, and if $A_{\max }^{n}$ is important then it has to be controlled when the monitor is constructed. The ratio of $A_{\min } / A_{\max }$ is also changing in time

$$
A_{\min } / A_{\max }^{n}=\phi_{\min }^{n} / \phi_{\max }^{n} .
$$

There is also a possibility to directly specify $A_{\max }$ and define $E^{n}$ as follows

$$
E^{n}=\phi_{\min }^{n} A_{\max } .
$$

In this case we will maintain the desired $A_{\max }$.

Let us now demonstrate meshes resulting from these strategies for 1D example of the Sod solution presented in previous sections. For our example, $\phi_{\max } \approx 21.2$ and $\phi_{\min } \approx 1.22$. The total integral of monitor function is equal to $\Phi \approx 2.44$.

Because we consider here a static mesh which corresponds to a given monitor, the difference in two strategies is just to how to define $E$ for $E=$ const, $E$ defined

\footnotetext{
3 "const" refers to constant in time
} 


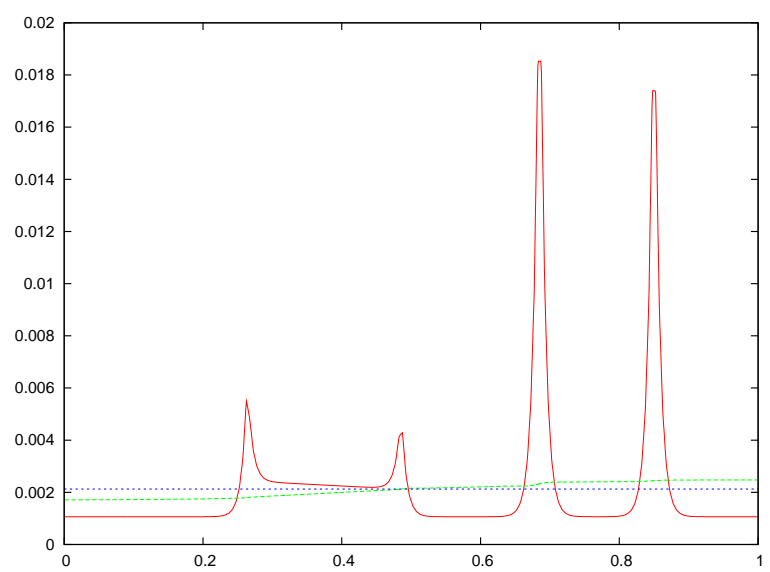

Figure 15: Sod problem: $E^{i n i}$ - red line, $E^{e q d}$ - green line, $E$ - blue line.

directly and for $A_{\min }=$ const, and $E$ computed from desired $h_{\min }$ and $\phi_{\max }$. For this reason we will show results only for A-ReALE $\left(A_{\text {min }}=\right.$ const $)$ strategy.

We will choose $h_{\min }=A_{\min }=0.0001$. This leads to $E=\phi_{\max } A_{\min } \approx$ 0.00212 and $N=\Phi / E \approx 1148$. The deduced value of $h_{\max } \approx 0.00174$.

Let us note that "equivalent" uniform mesh with the same spatial resolution will require $1 / 0.0001=10000$ cells $(1$ is the length of computational domain in this case), that is approximately 8.7 times more cells then adaptive mesh.

To construct a mesh satisfying the equi-distribution principle, we use the algorithm described in Section 2.4. In 1D according to equation $(16)(d=1)$, we need to set weight function equal to $\phi^{3}$ to achieve equi-distribution. In $1 \mathrm{D}$ we use a uniform background mesh and a uniform mesh with the number of cells equal to the number of needed generators as an initial guess.

In Fig.15 we present graphs of $E_{k}^{i n i}=h_{k}^{i n i} \phi_{k}^{i n i}$ and $E_{k}^{\text {eqd }}=h_{k}^{\text {eqd }} \phi_{k}^{\text {eqd }}$ as function of the coordinates. $E$ is the theoretical level of equi-distribution that we wish to achieve for a mesh satisfying the equi-distribution principle. This figure demonstrates that the final $E_{k}^{e q d}$ are very close to the theoretical equidistribution level $E$ (remember that theoretical results are obtained with assumption of an infinitely smoothed weight function and an infinite number of generators).

In Fig. 16 we show final mesh sizes $h_{k}^{\text {eqd }}$. This left panel of this figure demonstrates that the mesh is refined where we expect and $h_{\min }^{e q d}$ is very close to the prescribed value and reaches this value where monitor has its largest value. The maximum value of $h$ is little bit off at the right end of the domain because the equi-distribution is not perfect in this region. 


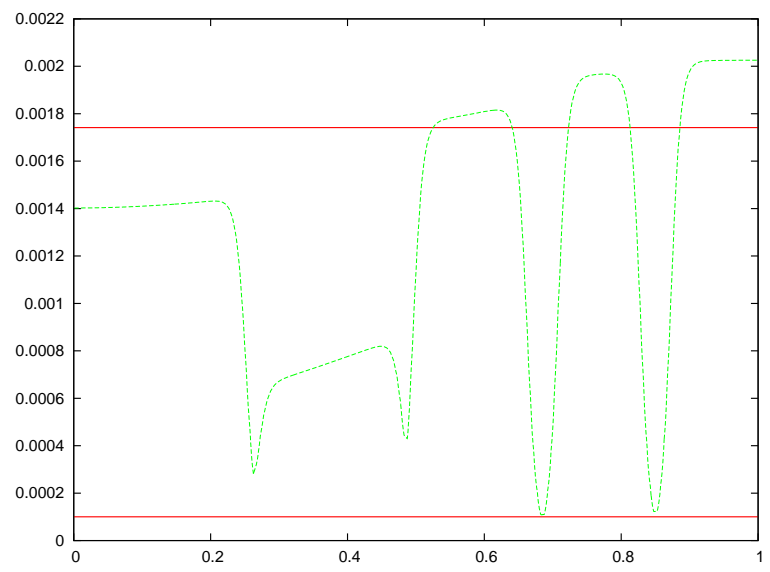

Figure 16: Sod problem: final mesh sizes $h_{k}^{e q d}$ as function of coordinates of cell center of the final mesh; solid lines in left panel are prescribed value of $h_{\min }$ and deduced value $h_{\max }$.

\section{ReALE Family of Methods}

In this section we will describe the reconnection-based ALE (ReALE) family of methods. We will start with description of the standard ReALE method introduced in [35]. Then, for completeness we will describe adaptive ReALE method: R-ReALE, (see, [5] for details) in which number of cells does not change but the mesh is adapting (by relocation of the generator's positions) to the monitor function according to the equi-distribution principle. Next, we will describe our new adaptive A-ReALE method, which is a fully adaptive method in which we add and remove cells and also move the generators to maintain specified spatial resolution and using the equi-distribution principle as described in previous section.

Let us mention that the main motivation for developing the original ReALE method was to create a robust method that would allow the computation of complex flows with large shear deformation without user intervention and while maintaining "Lagrangian" characteristics. The key to achieving these goals was to allow connectivity changes during the rezone stage of the method.

All three methods use the same Lagrangian phase that can be any method which can deal with arbitrary polygonal meshes. In [35] we present results for ReALE using both staggered and cell-centered discretizations. As one can find relevant references to Lagrangian discretizations in that paper; we will not repeat it here. In this paper our Lagrangian phase is based on cell-centered discretization introduced in [38, 37] and also briefly described in [35].

All three methods use an intersection-based remap [15, 18, 29]. The remapping phase is conceptually fairly simple. The quantities on the old Lagrangian mesh are cell-centered density, velocity and total energy that must be transferred 
on the rezoned mesh. First piecewise linear representations of cell-centered variables are constructed on the Lagrangian mesh. Then a slope limiting process, [3], is performed to enforce physically justified bounds. Conservative quantities, namely mass, momentum and total energy, are obtained by integration of these representations. New conservative quantities are calculated by integration over polygons of intersection of new (rezoned) and old (Lagrangian) meshes. Finally, primary variables are simply recovered by division by new volume (for density) or new mass (for momentum and energy). Clearly, because in general we have to remap between two meshes with different connectivity, we need to know with which cells of old mesh cell of new mesh has to be intersected. In our case it does not require global searches because we trace how generators which define new mesh are moving throughout the old mesh during adaptation process.

The real difference between the standard non-adaptive ReALE method and adaptive R-ReALE and A-ReALE methods is in the rezone phases. However, connectivity changes in both methods are performed using the machinery of the Voronoi diagrams. In this paper we only compare ReALE and A-ReALE, because comparison of ReALE and R-ReALE has been done in [5]. The comparison of the accuracy and performance of two methods will be demonstrated on numerical examples in the special section. Now we describe rezone stages for ReALE, R-ReALE and A-ReALE methods.

\subsection{Rezone phase of standard ReALE method}

In standard ReALE method described in [35] the number of generators does not change in time. At time $t^{n}$ the mesh is constructed using position of generators $\mathbf{G}_{i}^{n}$. That is, the Lagrangian step starts with mesh which is Voronoi mesh, which correspond to $\mathbf{G}_{i}^{n}$. We denote this mesh $L^{n}$. During the Lagrangian step, the generators play no role. The result of the Lagrangian phase is a mesh $L^{n+1}$ on which we have all flow data. Mesh $L^{n+1}$ is not a Voronoi mesh, but has the same connectivity as mesh $L^{n}$. The rezoned mesh $R^{n+1}$ is obtained as follows. First, one defines the "Lagrangian" position of generators $\mathbf{G}_{i}^{L, n+1}$ at $t^{n+1}$. These positions are obtain by moving generators from their positions $\mathbf{G}_{i}^{n}$ with some "Lagrangian" velocity averaged from vertices of the cell $i-\mathbf{u}_{i}^{L}$

$$
\mathbf{G}_{i}^{L, n+1}=\mathbf{G}_{i}^{n}+\mathbf{u}_{i}^{L} \Delta t .
$$

Then, one computes position of centroids $\mathbf{C}_{i}^{L, n+1}$ of the cell $i$ of the mesh $L^{n+1}$. Finally, the positions of generators $\mathbf{G}_{i}^{n+1}$ which will determine the rezoned mesh $R^{n+1}$ are defined as follows

$$
\mathbf{G}_{i}^{n+1}=\mathbf{G}_{i}^{L, n+1}+\omega_{i}\left(\mathbf{C}_{i}^{L, n+1}-\mathbf{G}_{i}^{L, n+1}\right), \quad 0 \leq \omega_{i} \leq 1,
$$

where the weight $\omega_{i}$ is based on eigenvalues of the deformation tensor for the cell $i$ and $\omega_{i}$ is zero under translation or solid rotation. There are several design principles for using (57) to define positions of the generators: first, it keeps mesh close to Lagrangian; second, it allows a smooth mesh to be maintained because $\mathbf{C}_{i}^{L, n+1}$ corresponds to one Lloyds iterations toward centroidal Voronoi 
mesh, which is a smooth mesh; and finally choice of the parameter $\omega_{i}$ allows us not to perturb the initial mesh until a time when the mesh starts to deform at particular location. The detail of the rezone stage for standard ReALE can be found in [35].

In the context of adaptation, we want to emphasize that the rezone strategy suggested in [35] keeps cells "Lagrangian" on average, that is shape and connectivity of the cells are changing but movement of the cell is "Lagrangian" on average. This is clearly demonstrated in [35] (Section 9). Therefore, standard ReALE has the adaptive properties of typical Lagrangian method. That is, it preserves an initial contact discontinuity and adapts to shock. However, it does not adapt to rarefaction and does not adapt to complicated flow structures that may appear during calculations, like interaction of the shock with contacts, see for example, [30].

\subsection{Rezone phase of adaptive R-ReALE method}

For R-ReALE the number of cell does not change in time. The monitor function at each time step is given by its value on mesh $L^{n+1}$. Initial mesh and mesh on each time in the rezone phase adapts to the current monitor according to the equi-distribution principle. The construction of the mesh that satisfies the equi-distribution principle is performed as described in previous sections of this paper.

The input data is the monitor function and number of generators $N$. On each time step it is reaches a different level of equi-distribution

$$
E(t)=\left(\int_{\Omega(t)} \phi(\mathbf{x}, t) d V\right) / N
$$

because the number of cells does not change. However, the global integral of the monitor function does change because the monitor and potentially the computational domain may change in time.

The detailed description of R-ReALE and its comparison with standard ReALE method has been done in [5].

\subsection{Rezone phase of adaptive A-ReALE methods}

The monitor function at each time step is given by its value on mesh $L^{n+1}$.

At the beginning of the $\mathrm{A}-\operatorname{ReALE}\left(A_{\min }=\right.$ const $)$ calculation, one need to choose $h_{\min }$ (and corresponding $A_{\text {min }}$ ), the required spatial resolution. This resolution will be maintained for entire calculation for cells in which the monitor reaches its maximum value. Next, one defines required level of equi-distribution according to equation

$$
E_{\text {rel }}=A_{\min } \max _{k} \phi_{k}
$$

and corresponding required number of cells

$$
N_{r e l}=\left(\sum_{i=1}^{N} \phi_{i}^{n}\left|\Omega_{i}\right|\right) / E_{r e l} .
$$


Now we can construct an adaptive mesh with $N_{\text {rel }}$ cells that satisfies equidistribution principle.

At the beginning of the $\mathrm{A}-\operatorname{ReALE}(E=$ const $)$ calculation, one need to choose $E_{r e l}$, the required equi-distribution level. This equi-distribution level will be maintained for entire calculation. Number of cells is computed using equation (59) and an adaptive mesh with $N_{\text {rel }}$ cells is constructed using equidistribution principle.

That is, the rezone phase for both adaptive ReALE methods differs only in how $E_{\text {rel }}$ is computed.

\section{How to compare ReALE and A-ReALE methods?}

The comparison of methods with the number of cells fixed in time as ReALE with A-ReALE is not a trivial task. We have chosen to compare different characteristics of the methods to obtain numerical solution for chosen final time $T$. Clearly one of most important characteristics is error at time $T$. However, one needs to consider the number of time steps to reach time $T$, the number of cells used in overall process (that is, sum of number of cells used on all time steps, denoted by $N_{s t}$ where the subscript "st" stands for space-time), the spatial resolution achieved, and so on. The most universal measure of the method is convergence rate. However, the investigation of convergence rate assumes some error model. The usual approach for Eulerian methods is to choose some parameter $h$ and use model of form $\varepsilon=C_{h} h^{q}+C_{t} \delta t$. For explicit methods $\delta t \sim h$, one can use the following model $\varepsilon=\tilde{C}_{h} h^{q}$. The choice of $h$ for Eulerian methods on uniform mesh is obvious. However, if one considers adaptive Eulerian methods that use adaptive mesh refinement, the choice of $h$ is not obvious. The situation is more complicated for pure Lagrangian methods because even though the number of cells in the initial mesh can be the same, the results strongly depend on choice of initial mesh. The situation with standard ALE methods is even more complicated because results depend on rezone strategy which may change depending on the resolution.

In A-ReALE there are several parameters. If parameters $\beta$ and $\alpha$, which participate in the raw monitor transformation are fixed, there is only one parameter $A_{m i n}$ for the A-ReALE $\left(A_{\min }=\right.$ const $)$ adaptation strategy or $E$ for A-ReALE $(E=$ const $)$ adaptation strategy. For A-ReALE $(E=$ const $)$, it is natural to choose $E$ as small parameter and investigate the convergence rate with respect to this small parameter. For $\operatorname{A}-\operatorname{Re} \operatorname{ALE}\left(A_{\text {min }}=\right.$ const $)$, it is natural to use $h_{\min }=\sqrt{A_{\min }}$ as $h$ in error model for this method. However, in the standard ReALE method, we cannot control $A_{\min }$ or $E$ and only parameter is $N$ so that it is natural to choose $N^{-1 / 2}$ as $h$ in the error model for ReALE. Therefore, the parameter $h$ has different meaning for ReALE and A-ReALE and one cannot compare the convergence orders of two methods using these models. Moreover, one also needs to decide upon the initial mesh. For A-ReALE, the initial mesh is adapted to initial data according to the monitor and equidistribution principle. It is almost obvious that such a mesh will not be optimal for the standard ReALE method and a uniform initial mesh can be better choice 
because it will adapt to the solution according to Lagrangian motion. In [4], the authors suggest using the cube root of total number of cells accumulated during entire run to reach time $T$ as parameter $h$. We are not sure that this is right thing to do and, for this reason, we do not compare convergence rates of ReALE and A-ReALE. However, we will present convergence rates for each method with respect its own $h$.

For both methods, we can compute some estimate of a norm of the spatial error at a particular time using either an exact solution (if it is known) or a reference solution obtained using high-resolution calculations. For example, we can compute $L 1$ error for density as follows

$$
e\left(t^{n}\right)=\int_{\Omega}\left|\rho\left(\mathbf{x}, t^{n}\right)-\rho_{e x t}\left(\mathbf{x}, t^{n}\right)\right| d \mathbf{x}=\sum_{i} \int_{\Omega_{i}}\left|\rho\left(\mathbf{x}, t^{n}\right)-\rho_{e x t}\left(\mathbf{x}, t^{n}\right)\right| d \mathbf{x}
$$

where $\rho_{\text {ext }}\left(x, t^{n}\right)$ is exact or reference solution and numerical solution $\rho\left(\mathbf{x}, t^{n}\right)$ is obtained from from $\rho_{i}^{n}$ by some piecewise linear reconstruction.

Any convergence analysis assumes some error model. In our case, it is natural to use the following model

$$
e(T)=C(T) h^{q(T)} .
$$

In this formula $h$ will be different for ReALE and A-ReALE, $q(T)$ is order of convergence and $C(T)$ is a constant that does not depend on the mesh. Let us mention that, in general, the order of convergence may depend on $T$. Again one needs to remember that $h$ has different meaning for different methods. To estimate "efficiency" one can compare how the number of generators changes with refinement for different methods.

Useful information for comparison of the ReALE and A-ReALE methods also can be obtained if one first runs A-ReALE method, computes an error and then chooses the number of cells in ReALE method such that error at final time is the same. Then one can compare the total number of needed generators over entire calculation as well as well as number of time steps needed to reach the final time. This information can be useful for analysis of relative efficiency of different methods. Some users may find it useful to know the number of cells in the so-called "equivalent" uniform mesh, that is, a mesh for which all cells have the same area equal to $A_{\min }$. If a particular problem has some spatial symmetry, like planar or cylindrical, then it can be useful to compare how well different methods preserve this symmetry.

For the test problems for which the error is difficult to obtain, we first run A-ReALE method and compute $N_{s t}$ and then calibrate the number of cells in ReALE method such that a similar $N_{s t}$ is obtained. This approach allows to compare the accuracy of two methods, when total number of generators in both method is similar.

In next Section we compare ReALE and A-ReALE methods with respect to the described metrics. 


\section{Numerical results}

In this section, we present several numerical examples that demonstrate performance of the new adaptive R-ReALE method in comparison with the standard non-adaptive ReALE method.

In Section 7.1 we present results for problem which has a smooth solution for a finite time and then forms a shock wave. This problem allows a numerical comparison of A-ReALE and ReALE methods for both smooth and discontinuous solutions. In Section 7.2 ,we present results for the classical Sedov point explosion. We solve a 1D cylindrically symmetric problem as a 2D problem in Cartesian $x, y$ coordinates. We then compare performance of ReALE and A-ReALE and show how errors for both methods depend on small parameter $h$ which defines the "resolution" of the method. In Section 7.3, we present results for the well known 2D "four shocks" Riemann problem, which is frequently used to test the performance of moving mesh methods [1, 49, 48]. In Section 7.4. we present a two material version of so-called "triple point" problem, [35]. This problem has been used in several papers, for example in [42] it was used to test performance of metric-based mesh adaptation. Finally, in Section 7.5 we present results for the problem of interaction between a planar shock wave and a square cavity, [25]. This flow has multiple shocks as well as region with high vorticity. We compare the simulation results from ReALE and A-ReALE with the experimental shadowgraphs. Both ReALE and and A-ReALE capture main features of the experimental data. As expected A-ReALE results are much more accurate than ReALE results.

We perform refinement study for all test cases. We use the subscript "c" and "f" to denote the quantity before and after mesh refinement. For A-ReALE $\left(A_{\min }=\mathrm{const}\right)$, we refine the mesh by reducing $A_{\min }$ by a factor of 4 , or $A_{\min , f}=A_{\min , c} / 4$; for A-ReALE (E=const), we refine the mesh by $E_{f}=E_{c} / 4$; for ReALE, we increase the number of generators $N_{f}=4 N_{c}$.

\section{1. "Smooth-to-shock" problem}

We consider a $1 \mathrm{D}$ flow of an ideal gas with $\gamma=3$ on a domain $[-9,9] \times$ $[-0.9,0.9]$. The initial states are

$$
\rho^{0}(x, y)=\frac{1}{\sqrt{\gamma}}\left(2+0.5 e^{-(x / 0.4)^{2}}\right), \quad p^{0}=\left(\rho^{0}\right)^{\gamma}, \quad \mathbf{u}^{0}=\mathbf{0} .
$$

The domain boundaries are treated as reflective walls. A smooth solution for the test problem exists until $t_{\text {shock }} \approx 0.932663$. After $t_{\text {shock}}$, the solution develops shock waves. Since the analytical solution can only be derived for $t<t_{\text {shock }}$ (see $[51,5]$ for details), we use a 1D Lagrangian code with 160000 cells to generate a reference solution. Figure 17 shows the density profile of the reference solution at $t=0,0.5,2$.

As a reference for A-ReALE methods, we use results obtained by standard ReALE method without adaptation. As we have discussed before, we start the ReALE simulations with a uniform mesh and because the velocity in the test 

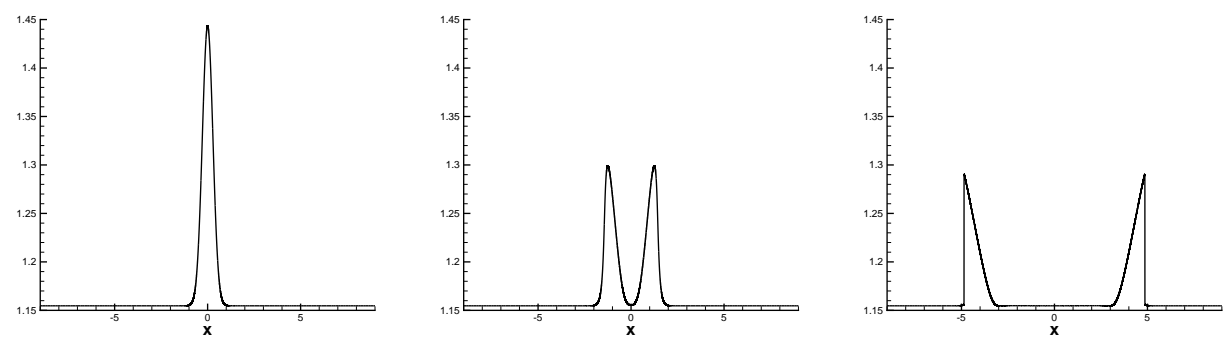

Figure 17: The density profile of the reference solution of the "Smooth-to-Shock" problem at $t=0,0.5,2$ (from left to right).
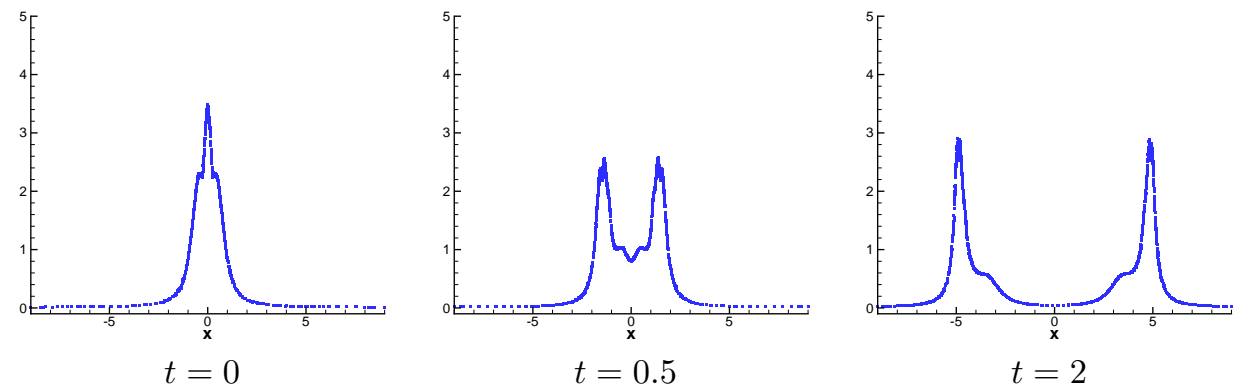

Figure 18: Smooth-to-shock problem. The scatter plot of the monitor function of the A$\operatorname{ReALE}\left(A_{\min }=\mathrm{const}\right)$ simulation with $A_{\min }=4 \times 10^{-3}$.

is small, the generators only move a small distance and the meshes are almost uniform for all the time steps - for this reason we do not show the mesh.

We perform four A-ReALE $\left(A_{\min }=\right.$ const $)$ simulations with $A_{\min }=4 \times$ $10^{-3}, 10^{-3}, 2.5 \times 10^{-4}$ and $6.25 \times 10^{-5}$. The monitor function is computed from density. The parameters are and $\alpha=1, \beta=0.9$. Figure 18 shows the monitor function from the simulation with $A_{\min }=4 \times 10^{-3}$ at $t=0,0.5,2$. Comparing Fig.17 and Fig.18, one can see that the monitor captures main features of the exact solution. The meshes for the corresponding time moments are shown in Fig.19. One can see that the monitor function controls the distribution of the cell area through the equi-distribution principle. Since the integral of the monitor function on each cell is approximately constant as it is suppose to be according to equi-distribution principle. In Fig.20, the area of the corresponding cell on a CCVT is approximately inversely proportional to the monitor function as it can be seen in Fig.21.

In this test case, we solve a $1 \mathrm{D}$ problem on a $2 \mathrm{D}$ domain, and the CCVT algorithm produces 2D meshes when used in 2D domain. Therefore, it is important to check how well the numerical algorithm preserves the 1D symmetry of the solution. In Figures 22 and 23, we plot the density isolines and the corre- 

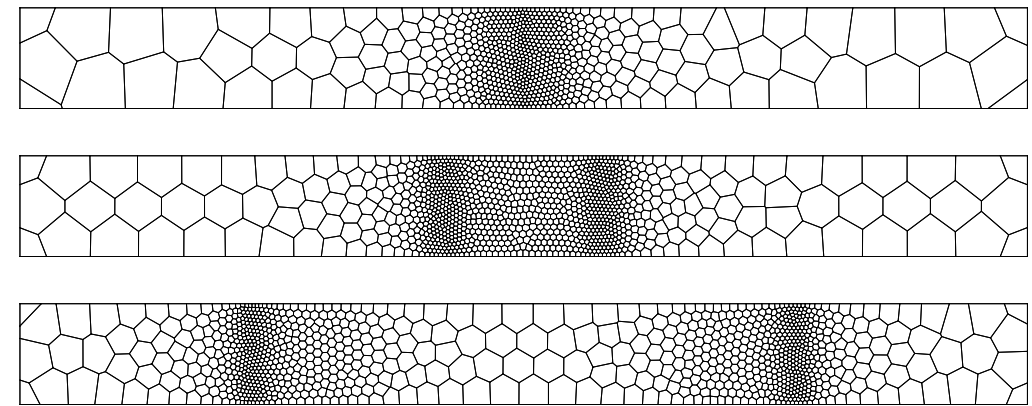

Figure 19: Smooth-to-shock problem. The meshes of the A-ReALE $\left(A_{\min }=\right.$ const $)$ simulation with $A_{\text {min }}=4 \times 10^{-3}: t=0,0.5,2$ from top to bottom.
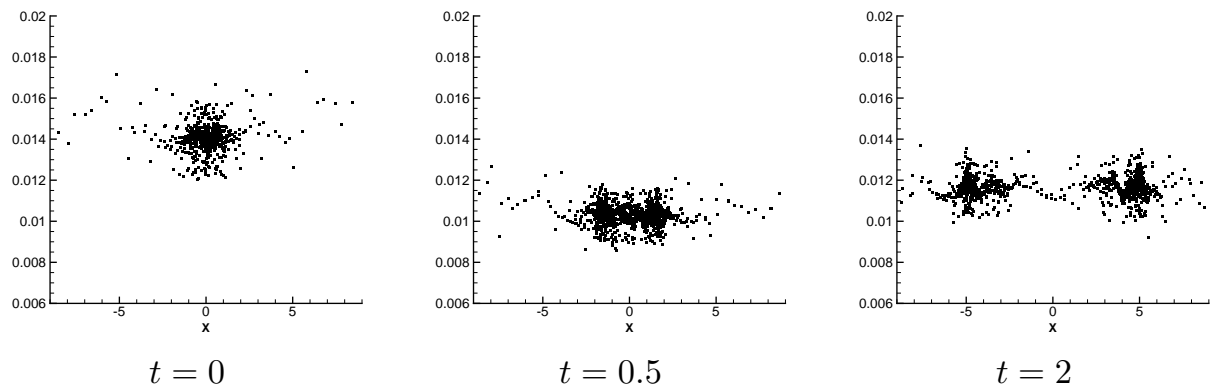

Figure 20: Smooth-to-shock problem. The scatter plot of the equi-distribution constant $E_{i}$ as function of $x$ from the $\mathrm{A}-\operatorname{ReALE}\left(A_{\min }=\right.$ const $)$ simulation with $A_{\text {min }}=4 \times 10^{-3}$.
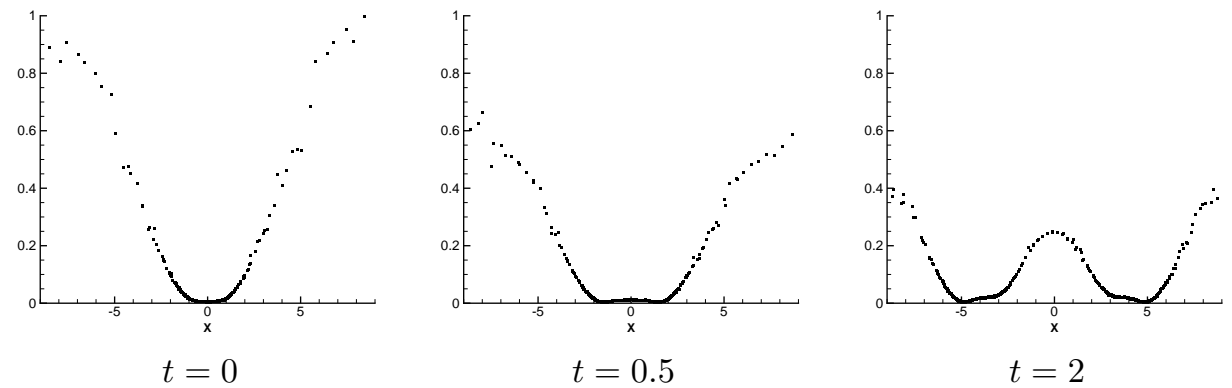

Figure 21: Smooth-to-shock problem. The scatter plot of the cell area from the A$\operatorname{ReALE}\left(A_{\min }=\mathrm{const}\right)$ simulation with $A_{\min }=4 \times 10^{-3}$. 

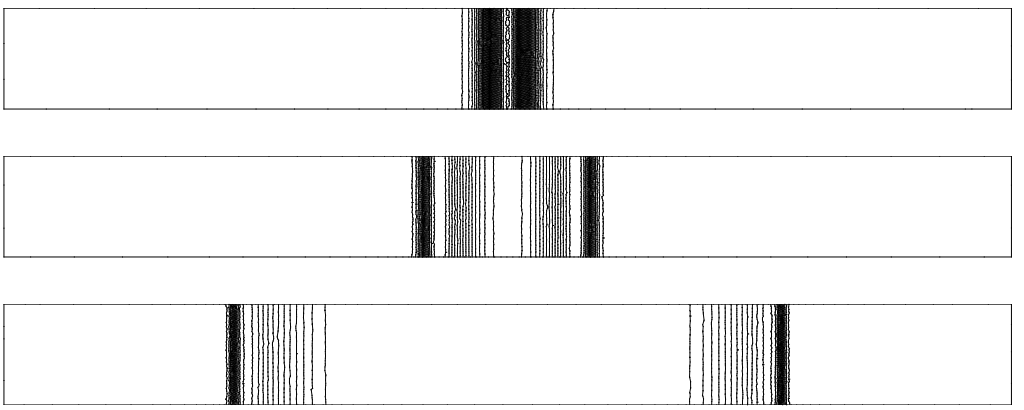

Figure 22: The density isolines from the A-ReALE $\left(A_{\min }=\right.$ const $): t=0,0.5,2$ from top to bottom.
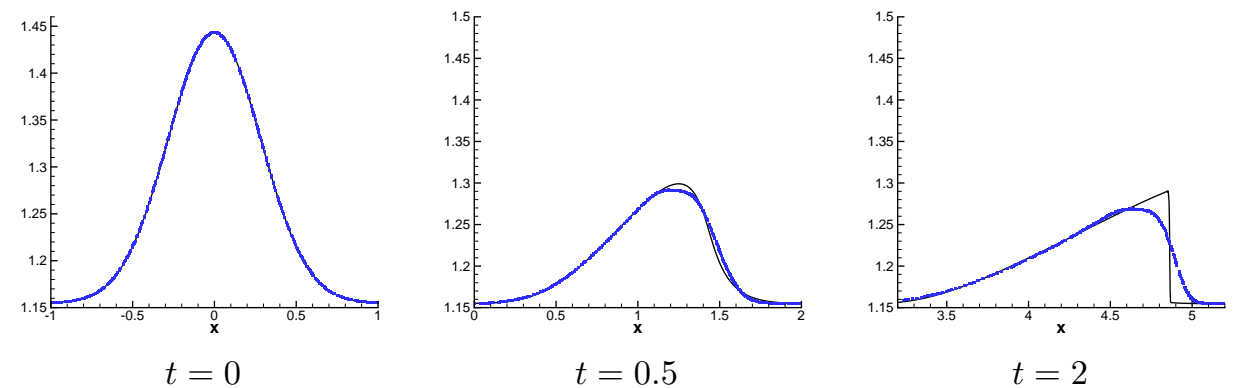

Figure 23: The scatter plot of density from A-ReALE $\left(A_{\min }=\right.$ const $)$ with using $A_{\min }=$ $4 \times 10^{-3}$. Black line: the reference solution. Blue dots: A-ReALE $\left(A_{\min }=\right.$ const $)$. For $t=0,0.5,1$ number of generators are 687,1137 and 1064 correspondingly.
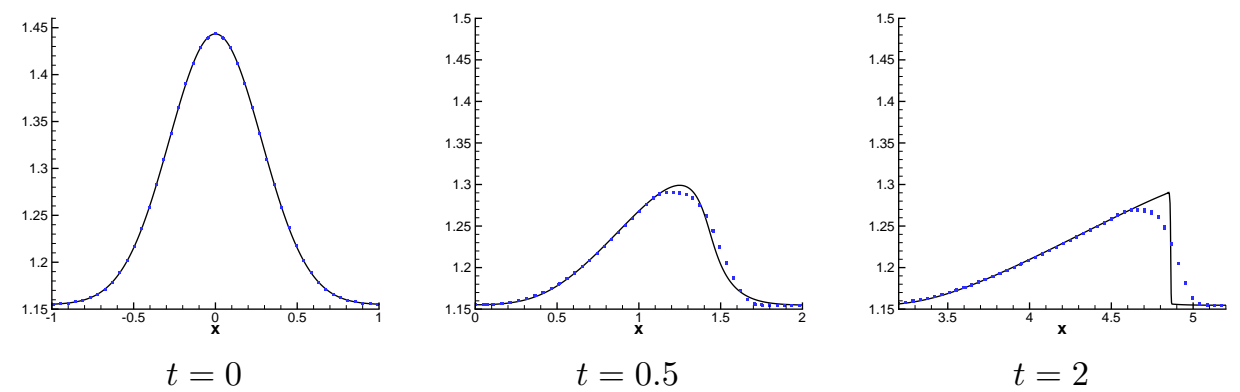

Figure 24: The scatter plot of density from ReALE with 4000 generators. Black line: the reference solution. Blue dots: $\mathrm{A}-\operatorname{ReALE}\left(A_{\min }=\right.$ const $)$. 
sponding 1D scatter plot. It can be seen that the 1D symmetry of the solution is well preserved. Figure 24 shows the scatter plot of density from ReALE with 4000 generators. Because the meshes are almost uniform for all time steps, the symmetry of the solution is also well preserved. In all panels in Fig. 24, the number of generators is the same and it is equal to 4000, standard ReALE method. In Fig. 23 for the A-ReALE method, the number of generators is changing and at $t=0$ is equal to 687 , at $t=0.5$ it is 1137 and at $t=2$ it is 1064. At all times, it is much smaller than for the ReALE method; however in the "visual" norm it has the same accuracy as ReALE. It will be confirmed later that A-ReALE is more accurate if both methods use same number of generators in the space-time domain.

For the A-ReALE $\left(A_{\min }=\right.$ const $)$ method, $A_{\min }$ is supposed to stay constant. Fig. 25 shows the history of $A_{\min }$ for A-ReALE $\left(A_{\min }=\right.$ const $)$. It can be seen that it is approximately constant, and it is at most $10 \%$ smaller than the given $A_{\text {min }}$.

To compare "efficiency" of standard ReALE and two A-ReALE methods it will be useful to analyze how number of generators depends on corresponding small parameter. In Fig.26, we show the number of generators for the two A-ReALE methods. The ratio of $N(t)$ for the two finest levels is presented in Fig.27. For $\mathrm{A}-\operatorname{ReALE}\left(A_{\min }=\right.$ const $)$, the ratio is around 4 at the early time. At late time, the ratio decreases and reaches 2 after the shock forms. For A-ReALE( $E=$ const $)$, the ratio is 2 for all the time steps.

Let us give some theoretical estimates for the number of generators that can explain these results. Given $\mathbf{x}$ at time $t$, if $\rho$ is smooth near $\mathbf{x}$, we have the following approximation of the monitor function

$$
\phi(\mathbf{x}, t) \approx \sqrt{\left\|H_{\rho}\right\|(\mathbf{x}, t)},
$$

where $\left\|H_{\rho}\right\|(\mathbf{x}, t)$ is the spectral norm of the Hessian matrix of $\rho$ at $(\mathbf{x}, t)$. If $\rho$ is discontinuous near $\mathbf{x}$, we have

$$
\phi(\mathbf{x}, t) \sim \frac{\sqrt{|\delta \rho|}}{h(\mathbf{x}, t)},
$$

where $h(\mathbf{x}, t)$ is the cell diameter at $(\mathbf{x}, t)$. If $\rho$ has both discontinuous and smooth part, we have

$$
\int_{\Omega} \phi d \mathbf{x}=\int_{\Omega_{1}} \phi d \mathbf{x}+\int_{\Omega_{2}} \phi d \mathbf{x},
$$

where $\Omega_{1}$ is a band covering the discontinuous part of $\rho$ and $\Omega_{1} \cup \Omega_{2}=\Omega$. If $\rho$ is discontinuous at $\mathbf{x}$, the thickness of the band is proportional to $h(\mathbf{x}, t)$ and

$$
\int_{\Omega_{1}} \phi d \mathbf{x}=\int_{\Omega_{1}} \frac{\sqrt{|\delta \rho|}}{h(\mathbf{x}, t)} d \mathbf{x} \sim \text { const. }
$$

On $\Omega_{2}$, we have

$$
\int_{\Omega_{2}} \phi d \mathbf{x}=\int_{\Omega_{2}} \sqrt{\left\|H_{\rho}\right\|}(\mathbf{x}, t) d \mathbf{x} \sim \text { const. }
$$


Thus

$$
\int_{\Omega} \phi d \mathbf{x} \sim \text { const, and }
$$

therefore,

$$
N(t)=\frac{\int_{\Omega} \phi d \mathbf{x}}{E(t)} \sim 1 / E(t) .
$$

For A-ReALE $(E=$ const $)$, we define refinement by reducing $E$ in half, and therefore, $E_{c}(t) / E_{f}(t)=2$ after each refinement and according to (64) we have

$$
N_{f}(t) / N_{c}(t) \approx 2 .
$$

For $\mathrm{A}-\operatorname{ReALE}\left(A_{\min }=\right.$ const $)$, there are two cases:

(i) $\rho$ is discontinuous in $\Omega$. From (63), we have

$$
\begin{aligned}
& E_{c}(t) / E_{f}(t)=\phi_{\max , c} A_{\min , c} /\left(\phi_{\max , f} A_{\min , f}\right)=h_{\min , f} A_{\min , c} /\left(h_{\min , c} A_{\min , f}\right) \\
& =\left(h_{\min , c} / 2\right) h_{\min , c}^{2} /\left(h_{\min , c}\left(h_{\min , c} / 4\right)\right)=2 .
\end{aligned}
$$

(ii) $\rho$ is smooth in $\Omega$. From (62), we have $\phi_{\max , c} \approx \phi_{\max , f}$ and

$$
E_{c}(t) / E_{f}(t)=\phi_{\max , c} A_{\min , c} /\left(\phi_{\max , f} A_{\min , f}\right)=A_{\min , c} / A_{\min , f}=4 .
$$

Therefore, for A-ReALE $\left(A_{\min }=\right.$ const $)$, we have the following estimate

$$
N_{f}(t) / N_{c}(t) \approx \begin{cases}2 & \text { if } \rho \text { is discontinuous in } \Omega \\ 4 & \text { if } \rho \text { is smooth in } \Omega\end{cases}
$$

The numerical results are close to these theoretical values in (65) and (67).

In Figure 28, we compare the L1 density error for the three methods. It can be seen $\mathrm{A}-\operatorname{ReALE}\left(A_{\text {min }}=\mathrm{const}\right)$ and $\mathrm{A}-\operatorname{ReALE}(\mathrm{E}=\mathrm{const})$ are more efficient than ReALE for shocks: at $t=2$, the error decreases by a factor of 2 after each refinement for all three methods. However, the number of generators increases by a factor of 4 for ReALE and by a factor of 2 for the two A-ReALE methods. In Table 3, we calibrate the number of generators of ReALE and $\mathrm{A}-\operatorname{ReALE}(\mathrm{E}=$ const $)$ so that both give similar L1 error as $\mathrm{A}-\operatorname{ReALE}\left(A_{\min }=\right.$ const) does at $t=0.5,2 . N_{s t}(t)$ is the total number of generators in space time domain, which can be used to compare the efficiency of different methods. One can see the two A-ReALE methods have similar accuracy and are more efficient than ReALE.

Table 4 shows the L1 density error of A-ReALE $\left(A_{\text {min }}=\right.$ const $)$ under mesh refinement. It is second order for smooth solution at $t=0.5$ and is first order for shocks at $t=2$. This is consistent with the numerical scheme we are using. Both the cell centered hydro solver and the conservative remap are second order for smooth solutions and first order for discontinuous solutions.

It maybe useful to consider the equivalent number of generators in a uniform mesh as for $\mathrm{A}-\operatorname{ReALE}\left(A_{\text {min }}=\right.$ const $)$ method

$$
N_{e q}=A / A_{\min },
$$




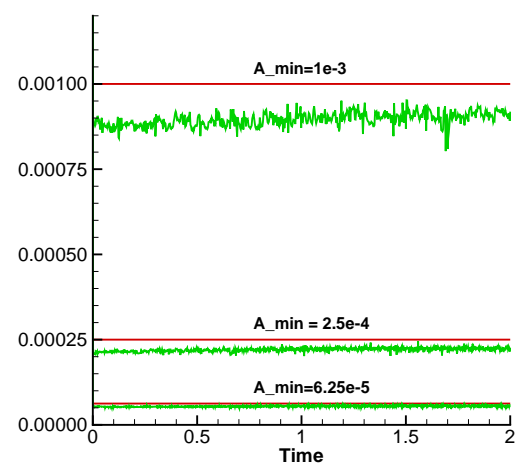

Figure 25: Smooth-to-shock problem. $A_{\min }$ for A-ReALE $\left(A_{\min }=\right.$ const $)$.

Table 3: Calibrating the number of generators for different simulations with respect to A$\operatorname{ReALE}\left(A_{\text {min }}=10^{-3}\right)$ results to reach the same L1 density error at time $t=0.5$ and $t=2$.

\begin{tabular}{|c|c|cccc|}
\hline & & $e(t)$ & $N$ & $N_{s t}(t)$ & time steps \\
\hline \multirow{3}{*}{$t=0.5$} & $\operatorname{ReALE}$ & $4.72185 \mathrm{e}-03$ & 20250 & 2792292 & 137 \\
& A-ReALE $\left(A_{\text {min }}=\right.$ const $)$ & $4.60268 \mathrm{e}-03$ & 3707 & 592182 & 172 \\
& A-ReALE $(\mathrm{E}=$ const $)$ & $4.74608 \mathrm{e}-03$ & 3726 & 557920 & 170 \\
\hline \multirow{3}{*}{$t=2$} & $\operatorname{ReALE}$ & $1.66233 \mathrm{e}-02$ & 20234 & 10339574 & 510 \\
& A-ReALE $\left(A_{\text {min }}=\right.$ const $)$ & $1.76991 \mathrm{e}-02$ & 2802 & 2041910 & 662 \\
& A-ReALE $(\mathrm{E}=$ const $)$ & $1.72606 \mathrm{e}-02$ & 3126 & 1892320 & 655 \\
\hline
\end{tabular}

where $A$ is the area of the domain. The results are given in Table 5. After mesh refinement, $N_{e q} / N(t)$ is around 10 at $t=0.5$ and increasing at $t=2$. We give the following explanation for this behavior. At $t=0.5$, the solution is smooth. From (67), it can be seen $N(t)$ increases by a factor of 4 when $A_{\text {min }}$ decreases by a factor of 4 . Thus, $N_{e q} / N(t)$ does not change after mesh refinement, which means $\mathrm{A}-\operatorname{ReALE}\left(A_{\min }=\right.$ const $)$ has the same efficiency as ReALE under mesh refinement for smooth solutions. At $t=2$, the solution is discontinuous. From (67), it can be seen that $N(t)$ increases by a factor 2 when $A_{\min }$ decreases by a factor of 4 . Thus, $N_{e q} / N(t)$ changes by the factor $4 / 2 \approx 2$ after mesh refinement which means $\operatorname{A}-\operatorname{ReALE}\left(A_{\min }=\right.$ const $)$ becomes more "efficient" on the fine mesh for shocks. From the above analysis, it can be seen $\mathrm{A}-\operatorname{ReALE}\left(A_{\min }=\mathrm{const}\right)$ and $\mathrm{A}-\operatorname{ReALE}(\mathrm{E}=\mathrm{const})$ have very similar efficiency. Therefore, we only compare ReALE and A-ReALE $\left(A_{m i n}=\right.$ const $)$ in the following numerical experiments.

\subsection{Sedov blastwave}

We consider the Sedov blastwave in a uniform medium. In the initial time, the total amount of released energy 1 is deposited in the center of the domain. 


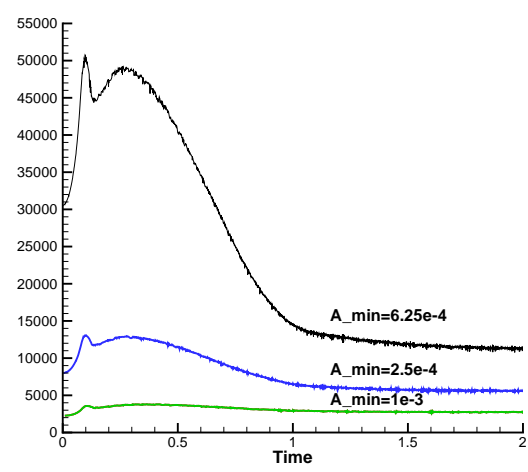

$\mathrm{A}-\operatorname{ReALE}\left(A_{\min }=\mathrm{const}\right)$

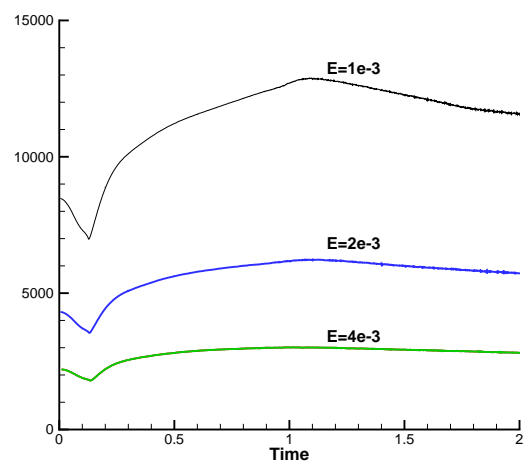

$\mathrm{A}-\operatorname{ReALE}(\mathrm{E}=\mathrm{const})$

Figure 26: Smooth-to-shock problem. Number of generators vs. time.

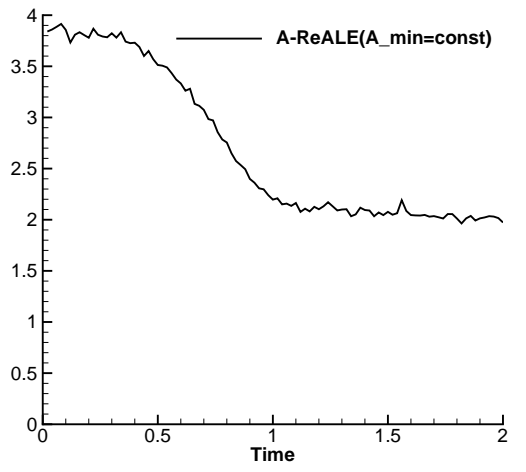

$\mathrm{A}-\operatorname{ReALE}\left(A_{m i n}=\right.$ const $)$

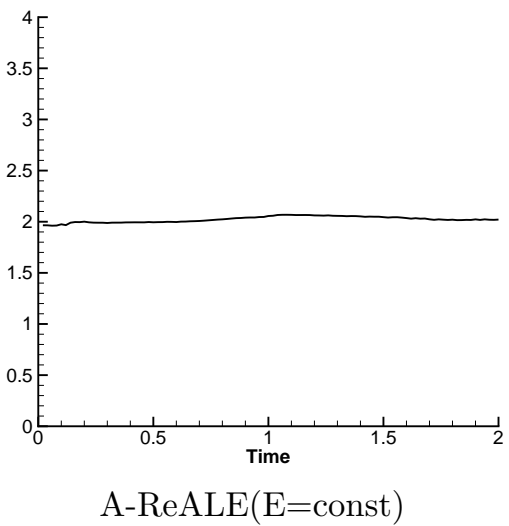

$\mathrm{A}-\operatorname{ReALE}(\mathrm{E}=\mathrm{const})$

Figure 27: Smooth-to-shock problem. The ratio of generator number $N_{f}(t) / N_{c}(t)$ vs. time. In the left panel, $N_{c}(t)$ and $N_{f}(t)$ are from the simulations with $A_{\min }=2.5 \times 10^{-4}$ and $6.25 \times 10^{-5}$. In the right panel, $N_{c}(t)$ and $N_{f}(t)$ are from the simulations with $E=2 \times 10^{-3}$ and $10^{-3}$

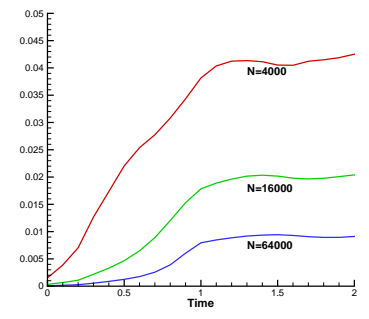

ReALE

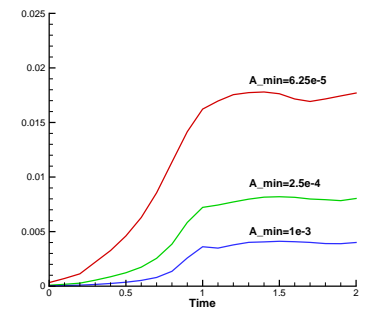

$\mathrm{A}-\operatorname{ReALE}\left(A_{\min }=\mathrm{const}\right)$

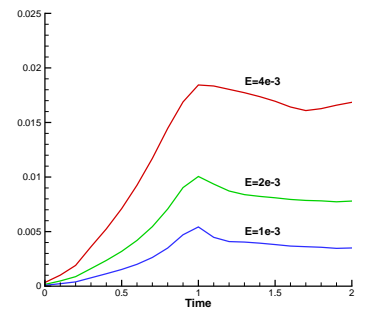

$\mathrm{A}-\operatorname{ReALE}(\mathrm{E}=\mathrm{const})$

Figure 28: Smooth-to-shock problem. L1 density error vs. time 
Table 4: Smooth-to-shock problem. L1 density error for A-ReALE $\left(A_{\min }=\right.$ const $)$.

\begin{tabular}{|c|ccc|}
\hline time & $A_{\min }$ & $e(t)$ & $q(t)$ \\
\hline \multirow{3}{*}{0.5} & $1 \mathrm{e}-3$ & $4.60268 \mathrm{e}-03$ & \\
& $2.5 \mathrm{e}-4$ & $1.23016 \mathrm{e}-03$ & 1.9036 \\
& $6.25 \mathrm{e}-5$ & $3.31996 \mathrm{e}-04$ & 1.8896 \\
\hline \multirow{3}{*}{2} & $1 \mathrm{e}-3$ & $1.76991 \mathrm{e}-02$ & \\
& $2.5 \mathrm{e}-3$ & $8.03586 \mathrm{e}-03$ & 1.1392 \\
& $6.25 \mathrm{e}-4$ & $4.00338 \mathrm{e}-03$ & 1.0052 \\
\hline
\end{tabular}

Table 5: Smooth-to-shock problem. The equivalent number of generators in a uniform mesh.

\begin{tabular}{|c|cccc|}
\hline time & $A_{\min }$ & $N_{e q}$ & $N(t)$ & $N_{e q} / N(t)$ \\
\hline \multirow{3}{*}{0.5} & $1 \mathrm{e}-3$ & 32400 & 3707 & 8.7402 \\
& $2.5 \mathrm{e}-4$ & 129600 & 11476 & 11.293 \\
& $6.25 \mathrm{e}-5$ & 518400 & 40314 & 12.859 \\
\hline \multirow{3}{*}{2} & $1 \mathrm{e}-3$ & 32400 & 2802 & 11.563 \\
& $2.5 \mathrm{e}-4$ & 129600 & 5581 & 23.222 \\
& $6.25 \mathrm{e}-5$ & 518400 & 11012 & 47.076 \\
\hline
\end{tabular}

The exact solution based on self-similarity arguments is available [28]. In numerical simulations, we deposit the released energy uniformly on a disk with radius 0.02 . The initial mesh is a uniform $\mathrm{VT}$ and the initial states are given by

$$
(\rho, e, \mathbf{u})=\left\{\begin{array}{cc}
\left(1,1 /\left(\pi r^{2}\right), \mathbf{0}\right) & r \leq 0.02 \\
\left(1,10^{-6}, \mathbf{0}\right) & r>0.02
\end{array},\right.
$$

where $r=\left(x^{2}+y^{2}\right)^{1 / 2}$. The final time of the simulation is $t=1$. The computational domain is a disk with radius 1.2 . To check the accuracy of the numerical solution, we compute a reference solution with 20000 cells using the code from [28]. See Figure 29 for the pressure of the reference solution at $t=1$.

To give a qualitative comparison of ReALE and A-ReALE, we first perform an A-ReALE simulation with $A_{\min }=1.6 \times 10^{-4}$ and a ReALE simulation with 4000 generators. As it will be shown later these are parameters for which ReALE and A-ReALE give approximately the same $L 1$ error at final time. The monitor function of A-ReALE is computed from pressure. The parameters are $\beta=0.9$ and $\alpha=1$. Figure 30 shows the monitor function from the A-ReALE simulation at $t=0$ and $t=1$. The corresponding meshes are shown in Figure 31. Because the shock is much more stronger at $t=0$ than at $t=1$ the maximum value of the monitor is large at $t=0$. It leads to the appearance of the big cells in the initial mesh. Also we have to put a lot of generators on the circular part of the external boundary to keep its integrity. The meshes from ReALE are given in Figure 32. It can be seen the cell areas are more uniform than those from A-ReALE at both $t=0$ and $t=1$. Figure 33 and 34 show the isolines and the 


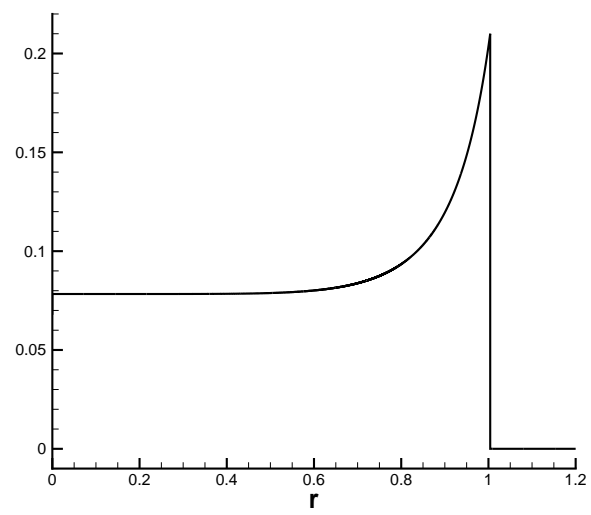

Figure 29: The pressure of the reference solution along radial direction at $t=1$.
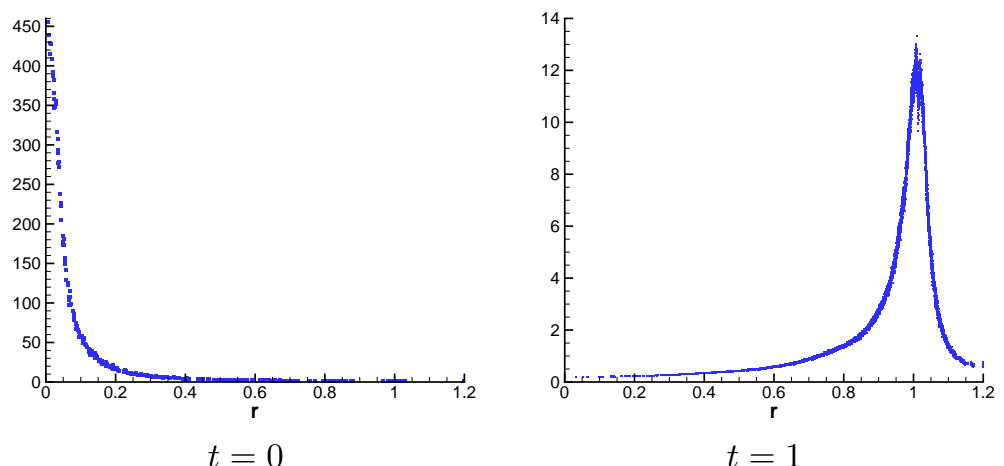

Figure 30: Sedov blastwave. The scatter plot of the monitor function along the radial direction from the simulation with $A_{\min }=1.6 \times 10^{-4}$.

scatter plot of pressure for ReALE and A-ReALE. The A-ReALE is certainly gives sharper profile at the shock front and it preserves symmetry as good as ReALE.

One can see that the symmetry of the solution near the shock front is better preserved in A-ReALE.

To compare performance of ReALE and A-ReALE, we show the simulation results for ReALE with 4000, 16000 and 64000 generators and A-ReALE with $A_{\min }=1.6 \times 10^{-4}, 4 \times 10^{-5}$ and $10^{-5}$. The left panel of Figure 35 shows the number of generators for A-ReALE. The right panel of Figure 35 shows the ratio $N_{f}(t) / N_{c}(t)$. It can be seen the ratio is about 2 which is consistent with the derivation in the previous section. Figure 36 compares the L1 density errors. We can give the following arguments to support statement that A-ReALE is more "efficient" than ReALE. After mesh refinement, the errors at $t=1$ decrease by 


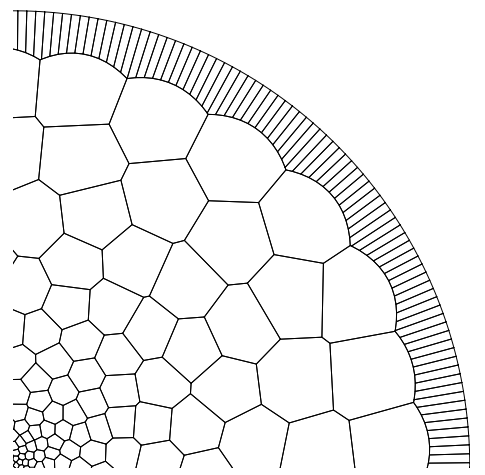

$t=0$

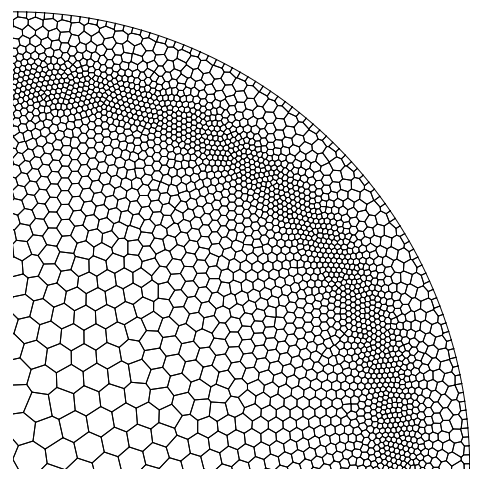

$t=1$

Figure 31: Sedov blastwave. The meshes of the A-ReALE simulation with $A_{\min }=1.6 \times 10^{-4}$.

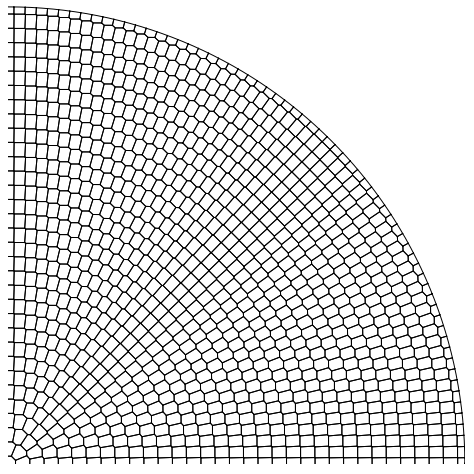

$t=0$

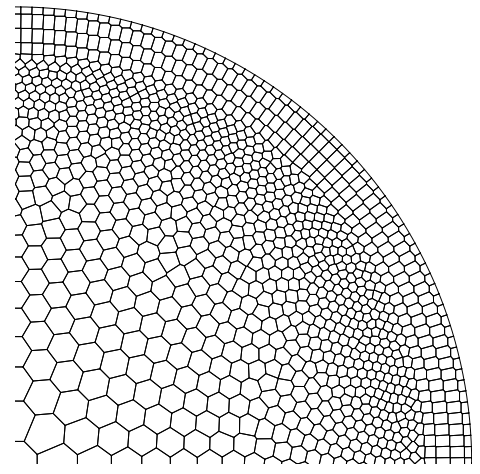

$t=1$

Figure 32: Sedov blastwave. The meshes of the ReALE simulation with 4000 generators. 

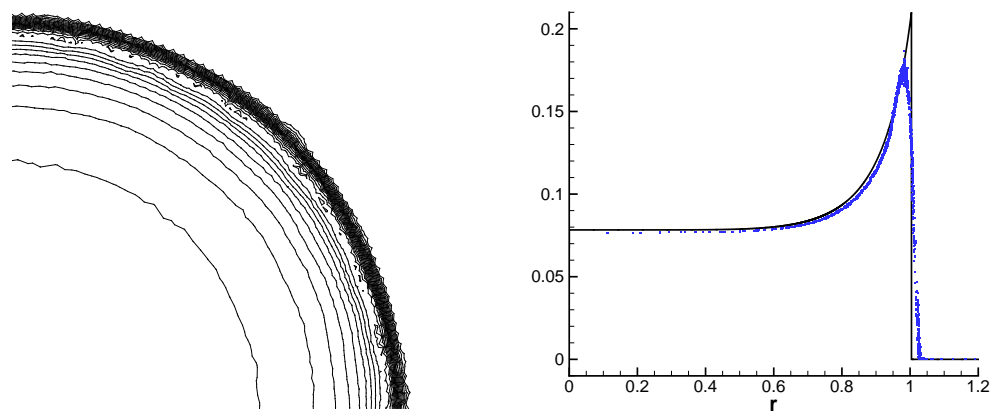

Figure 33: Sedov blastwave. ReALE with 4000 generators. Left: pressure isolines. Right: scatter plot of pressure along the radial direction.
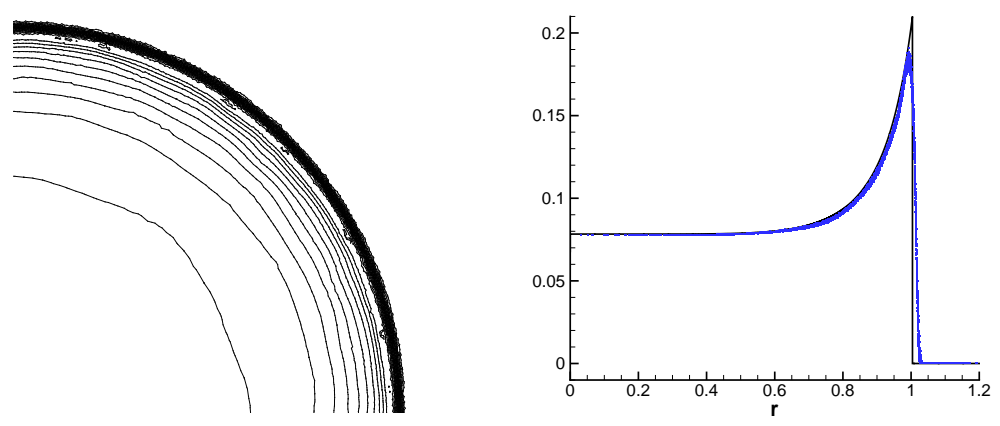

Figure 34: Sedov blastwave. A-ReALE with $A_{\min }=1.6 \times 10^{-4}$. Left: pressure isolines. Right: scatter plot of pressure along the radial direction. 

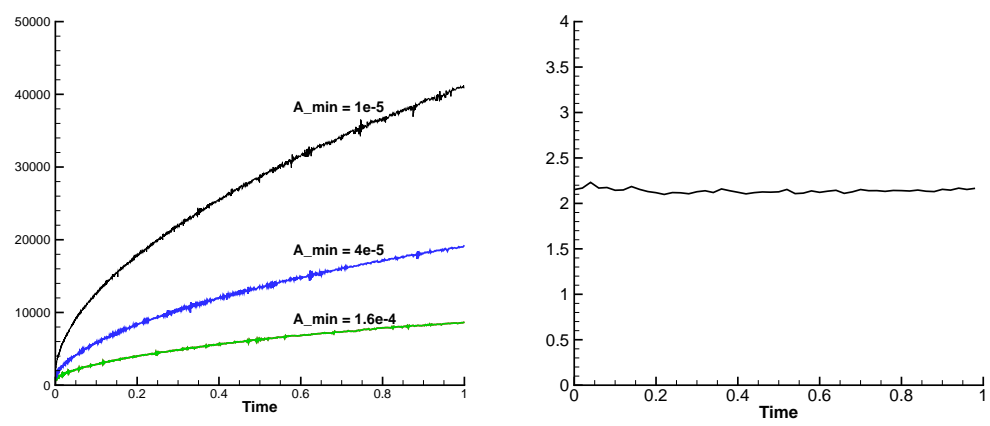

Figure 35: Sedov blastwave. Left: number of generators. Right: the ratio of the number of generators from the simulations of the two finest meshes.

Table 6: Sedov blastwave. L1 pressure error for A-ReALE.

\begin{tabular}{|ccc|}
\hline$A_{\min }$ & $e(1)$ & $q(1)$ \\
\hline $1.6 \mathrm{e}-4$ & $1.92016 \mathrm{e}-02$ & \\
$4 \mathrm{e}-5$ & $1.02941 \mathrm{e}-02$ & 0.89941 \\
$1 \mathrm{e}-5$ & $5.21725 \mathrm{e}-03$ & 0.98046 \\
\hline
\end{tabular}

a factor of 2 for both A-ReALE and ReALE. However, for ReALE, the number of generators increases by a factor of 4 after each refinement. For A-ReALE, the number of generators only increases by a factor of 2 after each refinement.

The errors at $t=1$ are given in Table 6 and 7 . One can see that both A-ReALE and ReALE show first order convergence, but remember that small parameters with respect to which convergence is established are different. Table 8 compares ReALE and A-ReALE simulations on the finest levels of refinement when both methods have similar L1 norm error. For this simulation $N_{s t}(1)$ (total number of generators in space-time to reach time $t=1$ ) of ReALE is 2 times more than for A-ReALE. In Fig.37, we show a zoomed view of the pressure color map for both methods. Although both methods have similar cell areas on the shock, A-ReALE generates larger cells away from the shock. In Fig.38 we compare the pressure along the radial direction. One can see that AReALE preserves symmetry better than ReALE does. The equivalent number of generators on a uniform mesh $N_{e q}$ and the ratio $N_{e q} / N$ are given in Table 9 . After refinement, the ratio $N_{e q} / N$ increases by a factor of 2 . This is consistent with the analysis for the number of generators in the A-ReALE method for discontinuous solutions presented in the previous section. 


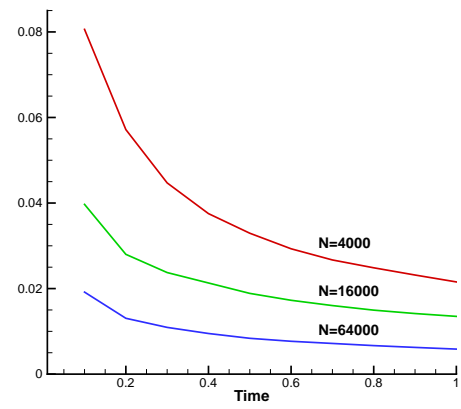

ReALE

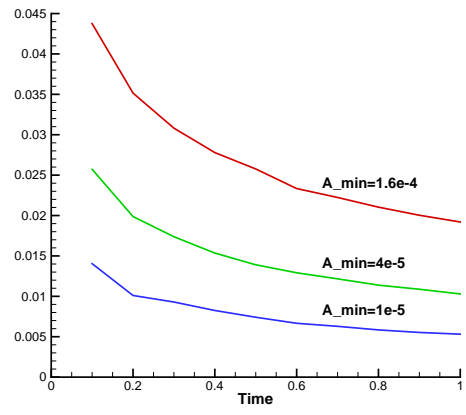

A-ReALE

Figure 36: Sedov blastwave. L1 density error vs. time

Table 7: Sedov blastwave. L1 pressure error for ReALE.

\begin{tabular}{|ccc|}
\hline$N$ & $e(1)$ & $q(1)$ \\
\hline 4000 & $2.15568 \mathrm{e}-02$ & \\
16000 & $1.34996 \mathrm{e}-02$ & 0.675 \\
64000 & $5.85117 \mathrm{e}-03$ & 1.206 \\
\hline
\end{tabular}

Table 8: Comparison of A-ReALE and ReALE simulations at $t=1$.

\begin{tabular}{|c|cccc|}
\hline & $e(1)$ & $N(1)$ & $N_{s t}(1)$ & time steps \\
\hline ReALE & $5.85117 \mathrm{e}-03$ & 64000 & $9.73 \mathrm{e} 7$ & 1716 \\
A-ReALE & $5.21725 \mathrm{e}-03$ & 40970 & $4.43 \mathrm{e} 7$ & 2107 \\
\hline
\end{tabular}

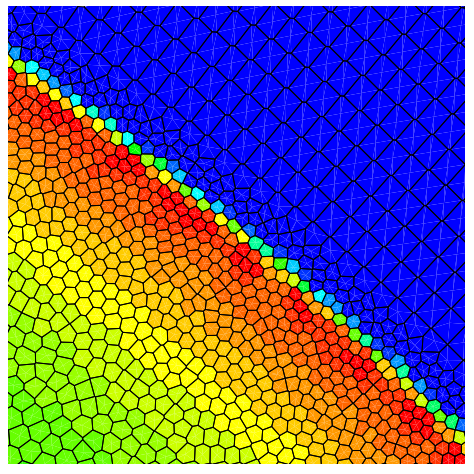

ReALE, 64000 generators

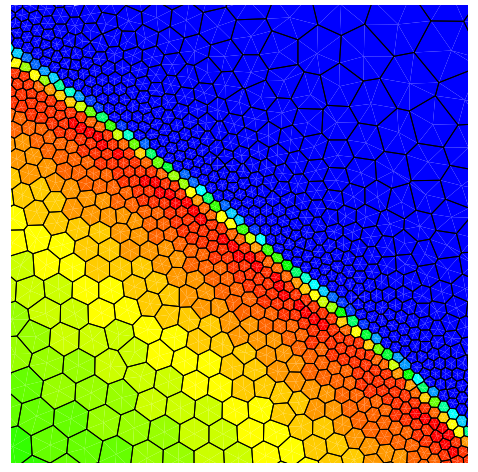

A-ReALE, $A_{\min }=10^{-5}$

Figure 37: Sedov blastwave. The zoomed view of mesh and pressure color map. The view window is $[0.56,0.7] \times[0.7,0.84]$ 


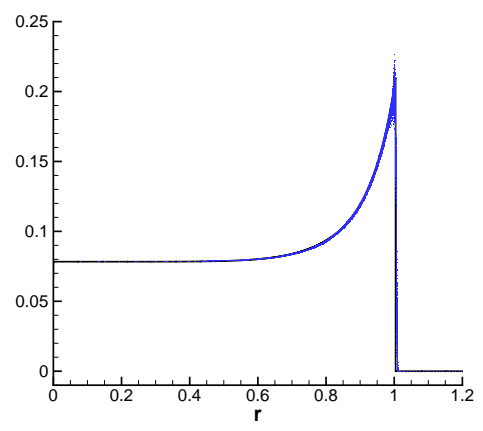

ReALE, 64000 generators

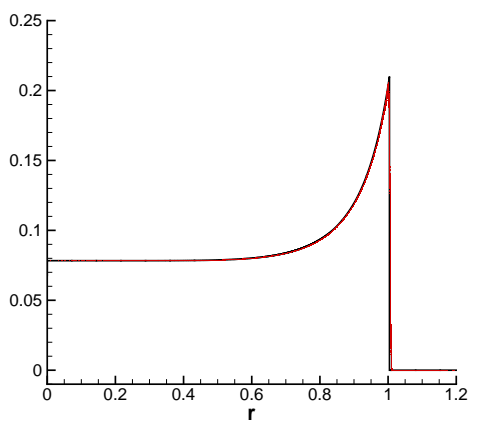

A-ReALE, $A_{\min }=10^{-5}$

Figure 38: Sedov blastwave. The scatter plot of pressure along the radial direction.

Table 9: Sedov blastwave. The equivalent number of generators in a uniform mesh.

\begin{tabular}{|cccc|}
\hline$A_{\min }$ & $N_{e q}$ & $N(1)$ & $N_{e q} / N(1)$ \\
\hline $1.6 \mathrm{e}-4$ & 28274 & 8557 & 3.3042 \\
$4 \mathrm{e}-5$ & 113096 & 19151 & 5.9055 \\
$1 \mathrm{e}-5$ & 452384 & 40970 & 11.042 \\
\hline
\end{tabular}

\subsection{A 2D Riemann problem}

We consider a four shock Riemann problem. The equation of state is an ideal gas with $\gamma=1.4$. The initial data are given as

$$
(\rho, p, u, v)= \begin{cases}(1.1,1.1,0,0) & x>0.5, y>0.5 \\ (0.5065,0.35,0.8939,0.0) & x<0.5, y>0.5 \\ (1.1,1.1,0.8939,0.8939) & x<0.5, y<0.5 \\ (0.5065,0.35,0.0,0.8939) & x>0.5, y<0.5\end{cases}
$$

which corresponds to the case of a left forward shock, a right backward shock, an upper backward shock, and a lower forward shock. This problem is frequently used to test the performance of moving mesh methods [1, 49, 48]. The computational domain is $[-0.223475,1] \times[-0.223475,1]$. The left and lower boundaries are the pistons moving with velocities which correspond to initial data. Reflection conditions are used for the right and upper boundaries. Final time is 0.25 .

We perform A-ReALE simulations with $A_{\min }=2 \times 10^{-5}, 5 \times 10^{-6}$, and $1.25 \times 10^{-6}$. The monitor function is computed from density. The parameters are $\beta=0.9$ and $\alpha=1$. In Fig.39, we show the initial mesh for A-ReALE with $A_{\text {min }}=2 \times 10^{-5}$. The initial mesh for ReALE is uniform with a similar number of generators. Figure 40 shows the mesh and density contour plots at $t=0.25$. One can see that the number of generators increases about 2 times after each refinement, which is consistent with the analysis in the previous section. Figure 


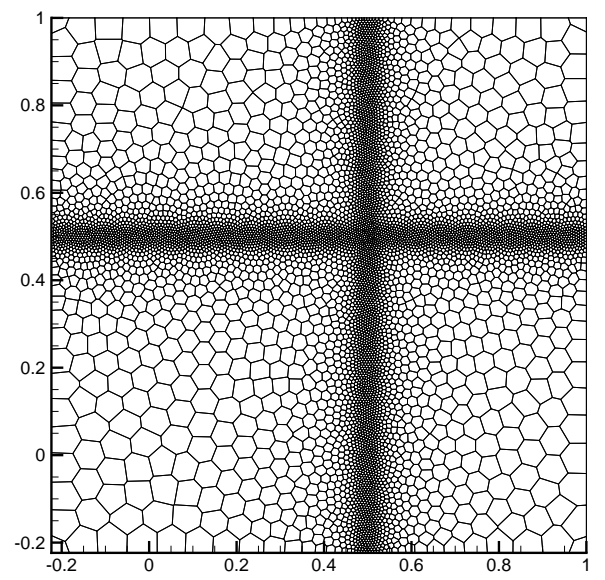

Figure 39: 2D Riemann problem. The initial mesh for A-ReALE simulation - $A_{\min }=2 \times 10^{-5}$, the number of generators is 6733 .

41 shows the results from a ReALE simulation with the same total number of generators as the finest A-ReALE simulation. It can be seen that A-ReALE has better resolution at the shocks than ReALE does. Although ReALE resolves the contact waves better, it generates numerical oscillations inside the shocked region - compare right panel in Fig.41 with pictures in right column in Fig.40. Figure 42 shows a zoomed view of the mesh and density contours near the upper left region of the domain. One can see that A-ReALE has smaller cells and better mesh quality than ReALE at the wave fronts.

\subsection{Single material triple point problem}

The computational domain is $[0,7] \times[0,3]$ which is composed of three regions denoted by $R_{1}=[0: 1] \times[0: 3], R_{2}=[1: 7] \times[0: 1.5]$ and $R_{3}=[1: 7] \times[1.5: 3]$. The fluid is an ideal gas law with $\gamma=1.4$. The initial data are given as

$$
(\rho, p, u, v)= \begin{cases}(1,1,0,0) & (x, y) \in R_{1} \\ (1,0.125,0,0) & (x, y) \in R_{2} \\ (0.1,0.125,0,0) & (x, y) \in R_{3}\end{cases}
$$

Reflection conditions are used for all the boundaries.

We perform A-ReALE simulations with $A_{\min }=8 \times 10^{-4}, 2 \times 10^{-4}$, and $5 \times$ $10^{-5}$. The monitor function is computed from internal energy. The parameters are $\beta=0.9$ and $\alpha=1$. Figure 43 shows the initial mesh of A-ReALE with $A_{\min }=8 \times 10^{-4}$. To make a direct comparison for A-ReALE and ReALE, we perform a ReALE simulation with 33556 generators. The total number of generators for this ReALE simulation is similar to that for the A-ReALE simulation with $A_{\min }=5 \times 10^{-5}$. Figure 44 shows the mesh and internal energy contour plots at $t=3$. Figure 45 shows the results from the ReALE 

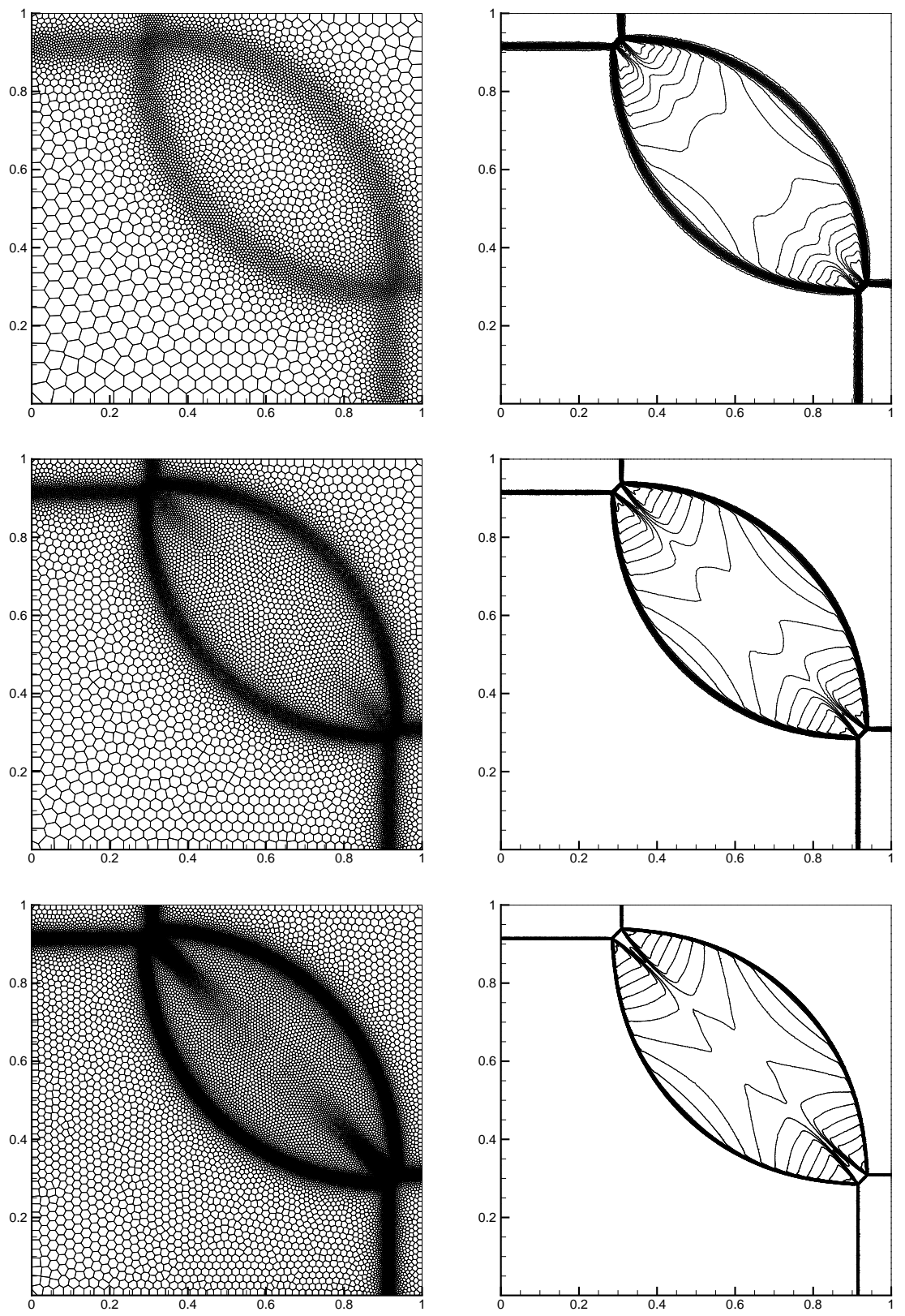

Figure 40: Visual convergence - the mesh and the density contours. A-ReALE calculations with $A_{\min }=2 \times 10^{-5}, 5 \times 10^{-6}, 1.25 \times 10^{-6}$. The number of generators at $t=0.25$ is 6616 , 14815, 32897. The total numbers of generators are 3799651, 17220452 and 75811305. 

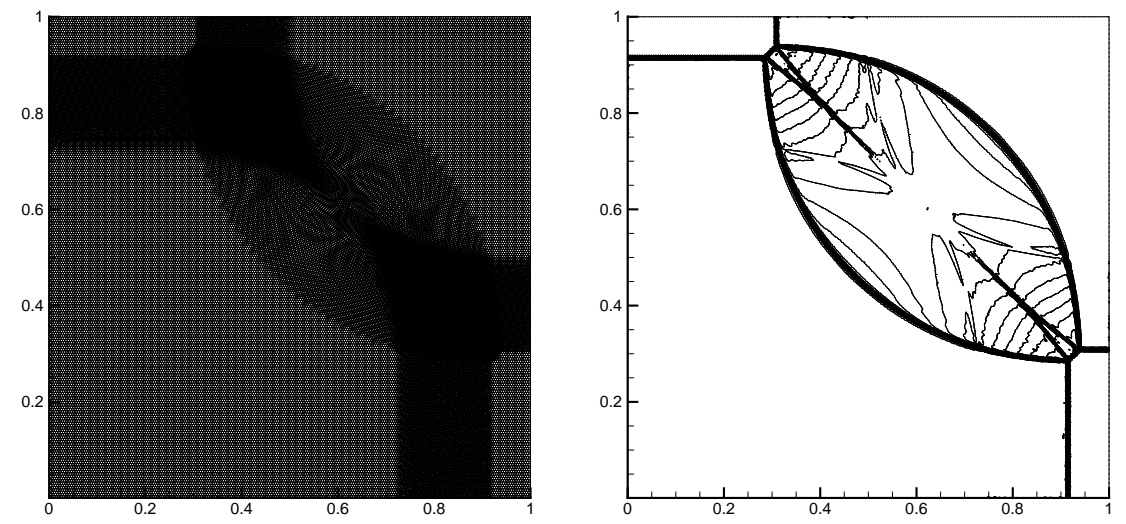

Figure 41: The mesh and the density contours of the ReALE simulations. The number of generators is 64070 . The total numbers of generators is 74753840 .

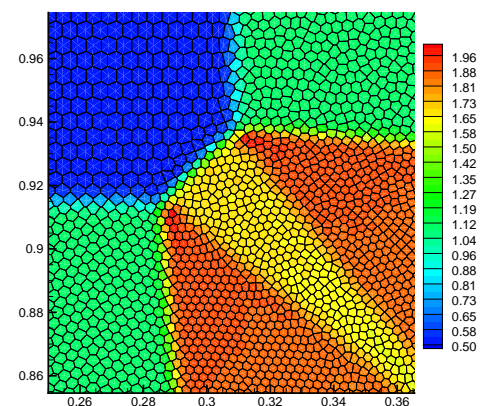

ReALE with 64070 generators

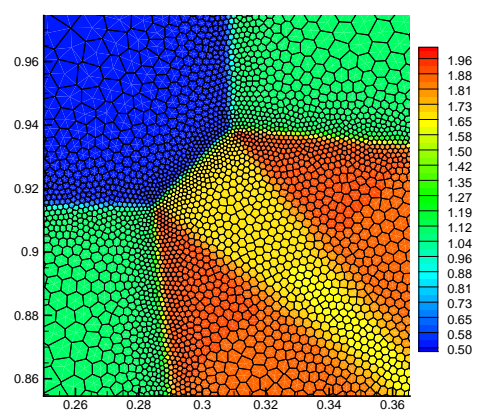

A-ReALE with $A_{\min }=1.25 \times 10^{-6}$

Figure 42: A 2D Riemann problem. The zoomed view of mesh and density contour at $t=0.25$. 


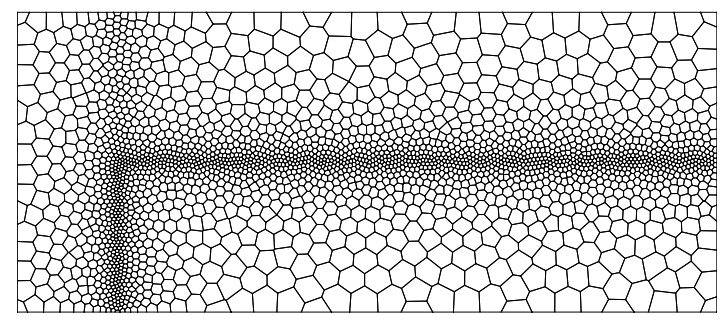

Figure 43: Triple point problem. The initial mesh for A-ReALE simulation - $A_{\text {min }}=8 \times 10^{-4}$, the number of generator is 2406 .

simulation at $t=3$. One can see that the A-ReALE coarsens the mesh between the contact and the shock and refines the mesh at the vortex. For ReALE, the mesh between the contact and the shock is finer than that on the vortex. This leads to poor resolution at the vortex and unnecessarily high resolution in the unimportant region. Figure 46 and 47 show the results at $t=5$. One can see A-ReALE with $A_{\min }=5 \times 10^{-5}$ gives more detail at the vortex.

In the left panel of Fig.48, we superimpose the ReALE mesh corresponding to bottom left picture in Fig.46 with the color map for specific internal energy. A similar picture for A-ReALE which corresponds to the bottom left picture in Fig.46 is presented in the right panel of Fig.48

Figure 49 gives a zoomed view of the mesh and the internal energy on the vortex. The mesh from the A-ReALE simulation is much finer than that from the ReALE simulation.

\subsection{Interaction between a shock and cavity.}

We focus on the interaction between a planar shock wave and a square cavity. This test case was first introduced in [25]. A numerical study using ReALE was performed in [21]. The computational domain is shown in Fig.50. Inside the computation domain, the initial states are

$$
(\rho, p, u, v)=\left\{\begin{array}{cc}
\left(1.73 \times 10^{-3}, 1.75,15.3,0\right) & x<-0.575 \\
\left(1.14 \times 10^{-3}, 0.97,0,0\right) & x \geq-0.575
\end{array},\right.
$$

which correspond to an incident shock with Mach number $M_{s}=1.3$. The equation of state is an ideal gas with $\gamma=1.4$. On the left-most boundary, a piston condition which maintains the shock is used. All other boundaries are reflective walls. For the A-ReALE simulations, the monitor function is computed from density and the parameters are $\beta=0.9$ and $\alpha=1$.

We compare the simulation results from ReALE and A-ReALE with the shadowgraphs presented in [25]. In order to make a direct comparison of ReALE and A-ReALE, we perform four simulations. The parameters, number of generators, total number of generators in the space time domain and time steps are given in Table 10. One can see that ReALE $N=6051$ and A-ReALE 

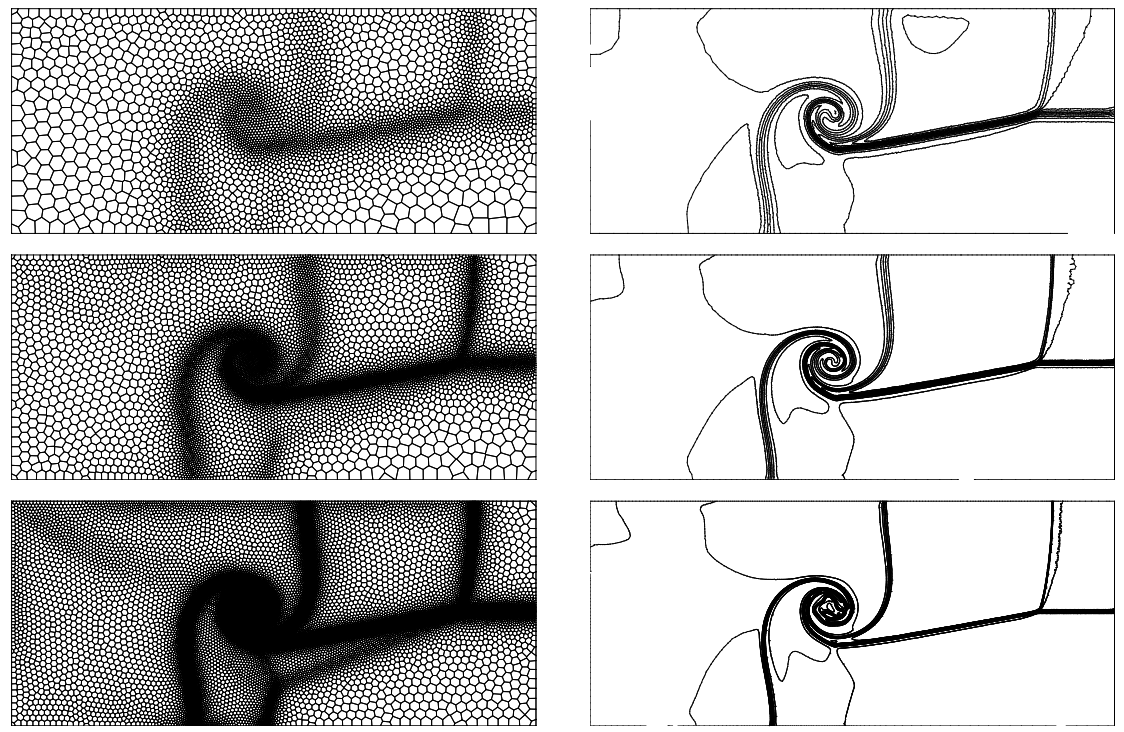

Figure 44: Triple point problem. Visual convergence at $t=3$ - the mesh and the internal energy contours. A-ReALE calculations with $A_{\min }=8 \times 10^{-4}, 2 \times 10^{-4}, 5 \times 10^{-5}$. The number of generators is $3669,9398,24374$.
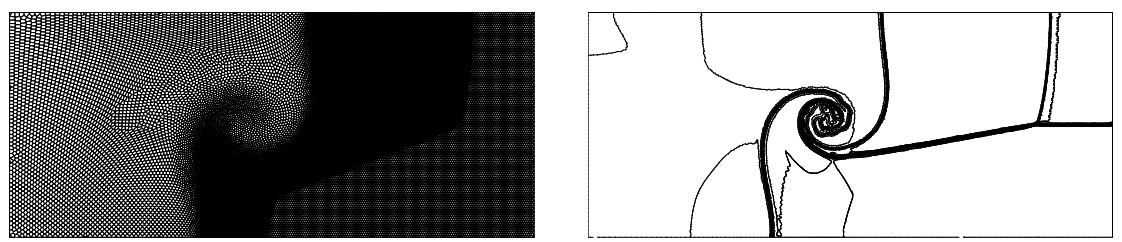

Figure 45: Triple point problem. The mesh and the internal energy contours of the ReALE simulations. The number of generators is 33556 . 

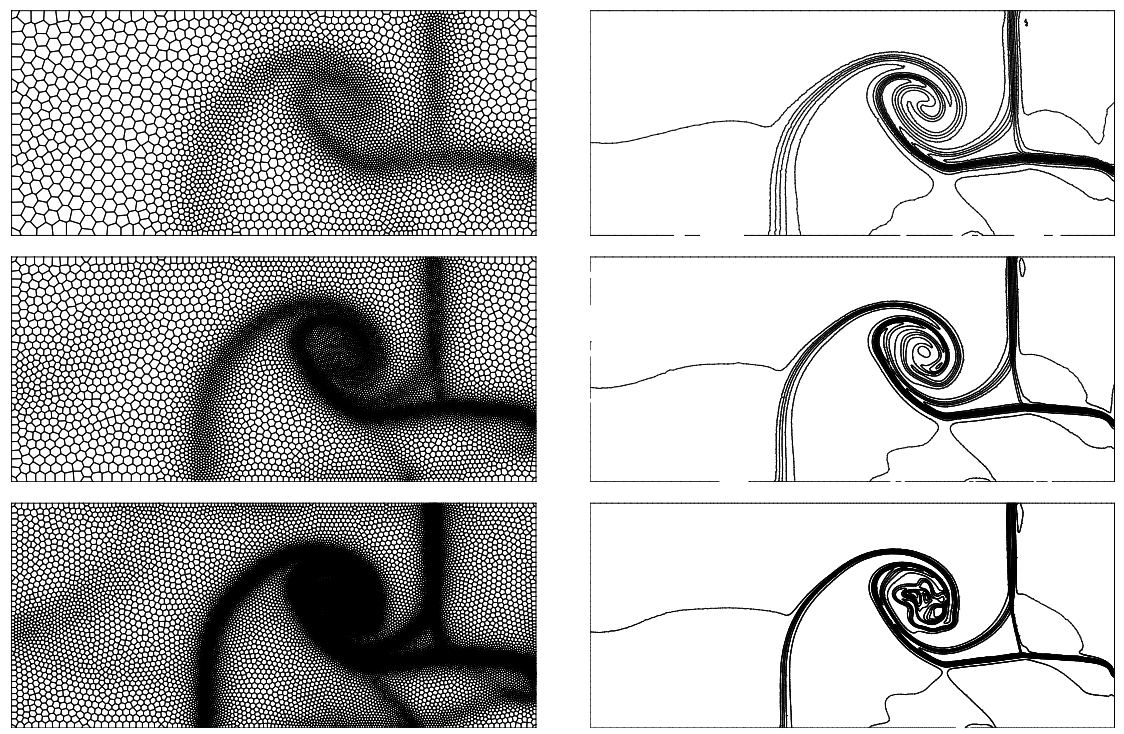

Figure 46: Triple point problem. Visual convergence at $t=5$ - the mesh and the internal energy contours. A-ReALE calculations with $A_{\min }=8 \times 10^{-4}, 2 \times 10^{-4}, 5 \times 10^{-5}$. The number of generators is $4342,9604,22330$.
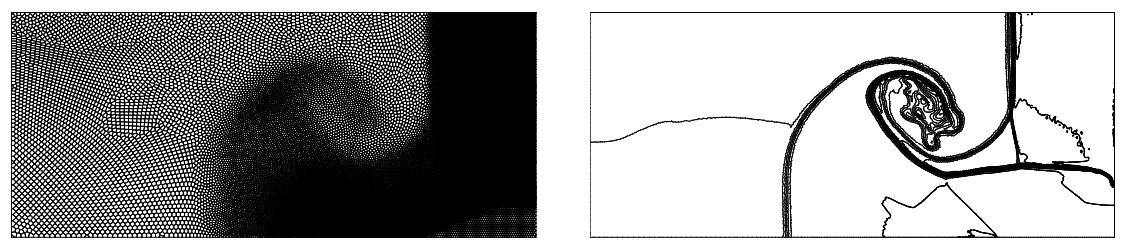

Figure 47: Triple point problem. The mesh and the internal energy contours of the ReALE simulations. The number of generators is 33556 . 

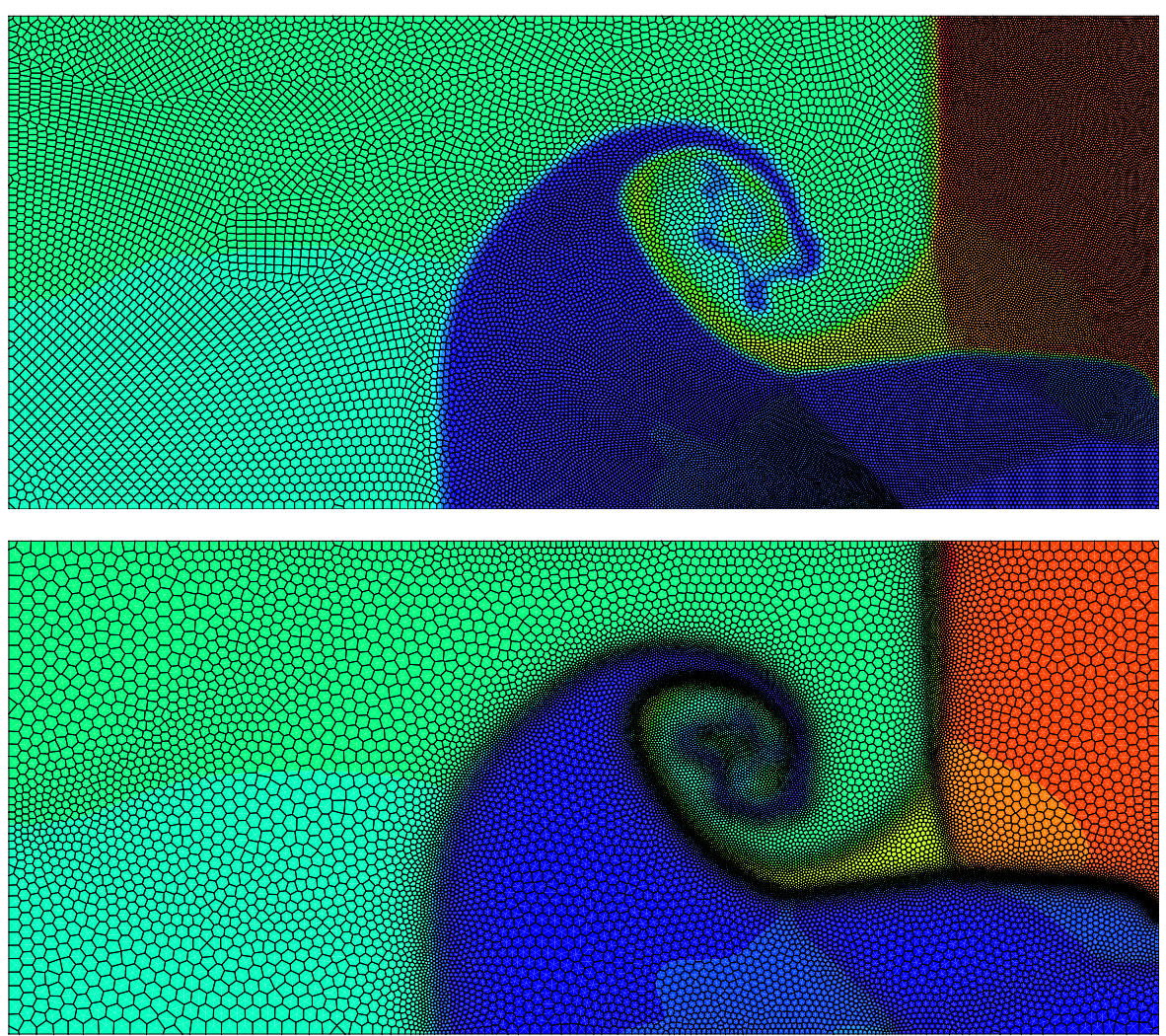

Figure 48: Triple point problem. Top - ReALE with 33556 generators; bottom - A-ReALE with $A_{\min }=5 \times 10^{-5}$ The mesh and internal energy color map at $t=5$.

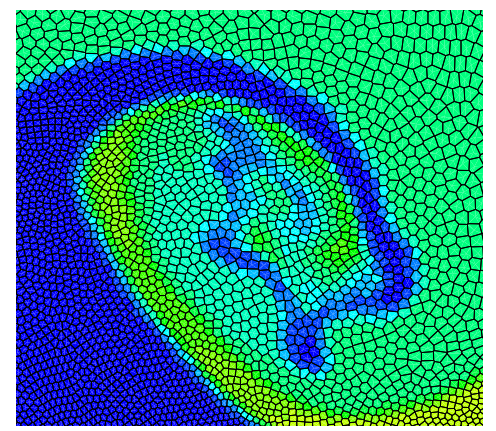

ReALE with 33556 generators

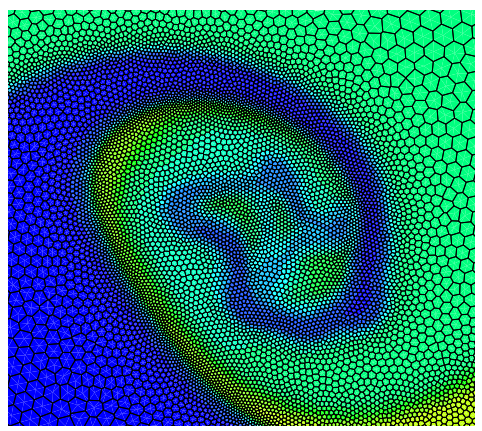

A-ReALE with $A_{\min }=5 \times 10^{-5}$

Figure 49: Triple point problem. The zoomed view of mesh and internal energy contours at $t=5$. 
Table 10: Interaction between a shock and cavity. Number of generators and time steps for ReALE and A-ReALE simulations. $N(420)$ : number of generators at $t=420 . N_{s t}(420)$ : total number of generators in the space time domain at $t=420$.

\begin{tabular}{|c|ccc|}
\hline & $N(420)$ & $N_{s t}(420)$ & time steps \\
\hline ReALE $N=6051$ & 6051 & 8707389 & 1439 \\
A-ReALE $A_{\min }=2 \times 10^{-3}$ & 6450 & 10684545 & 2212 \\
\hline ReALE $N=39593$ & 39593 & 159559790 & 4030 \\
A-ReALE $A_{\min }=1.25 \times 10^{-4}$ & 33179 & 177856789 & 7086 \\
\hline
\end{tabular}

$A_{\text {min }}=2 \times 10^{-3}$, ReALE $N=39593$ and A-ReALE $A_{m i n}=1.25 \times 10^{-4}$ use similar total numbers of generators in the space time domain. The initial meshes for ReALE $N=6051$ and A-ReALE $A_{\min }=2 \times 10^{-3}$ are given in Figure 50 .

Figure 51 shows the mesh and pressure contour plots at $t=160$. The diffracting shock is reflected by the wall of the cavity and forms three reflected shocks. The incident shock $S_{1}$ is strongest among all the shocks. A vortex forms near the upper-left corner of the cavity. From the left column of Figure 51, one can see that the mesh from A-ReALE has smaller cells than ReALE does at the shocks. One major difference between the two simulations is that A-ReALE has much better resolution at the vortex. This is because in the ReALE simulation, the diffracting shock moves generators into the shocked regions, which makes the mesh coarser on the vortex. For the A-ReALE simulation, the large density variation of vortex is detected by the monitor function and more generators are placed on the vortex. Figure 52 and 53 compares meshes and the pressure contour plots at $t=340$ and 420 . One can observe that the size of the vortex has increased while its center has slowly moved to the right of the cavity. It appears that that with same total number of generators, A-ReALE better resolves flow features. A-ReALE with $A_{\min }=2 \times 10^{-3}$ resolves the vortex even better than ReALE $N=39593$.

Both ReALE and A-ReALE numerical results are in a good agreement with the shadowgraphs from the experiment. This numerical experiment constitutes a validation of both methods by demonstrating their capabilities of producing physically accurate simulations of complex shock wave structures.

\section{Conclusion and future work}

We have described a new adaptive reconnection-based arbitrary LagrangianEulerian method, A-ReALE. The A-ReALE method uses a monitor function as an indicator of the error. The raw monitor function is smoothed to guarantee gradual changes in cell size and scaled to avoid very small and very big cells. We use an equi-distribution principle for the monitor function and centroidal Voronoi tessellation machinery as a tool to create the adaptive mesh.

In the A-ReALE $\left(A_{\text {min }}=\right.$ const $)$ method, the number of mesh cells changes in time to maintain a chosen spatial resolution in the cells where the monitor function is reaches its maxima. In the A-ReALE( $E=$ const $)$ method, the 

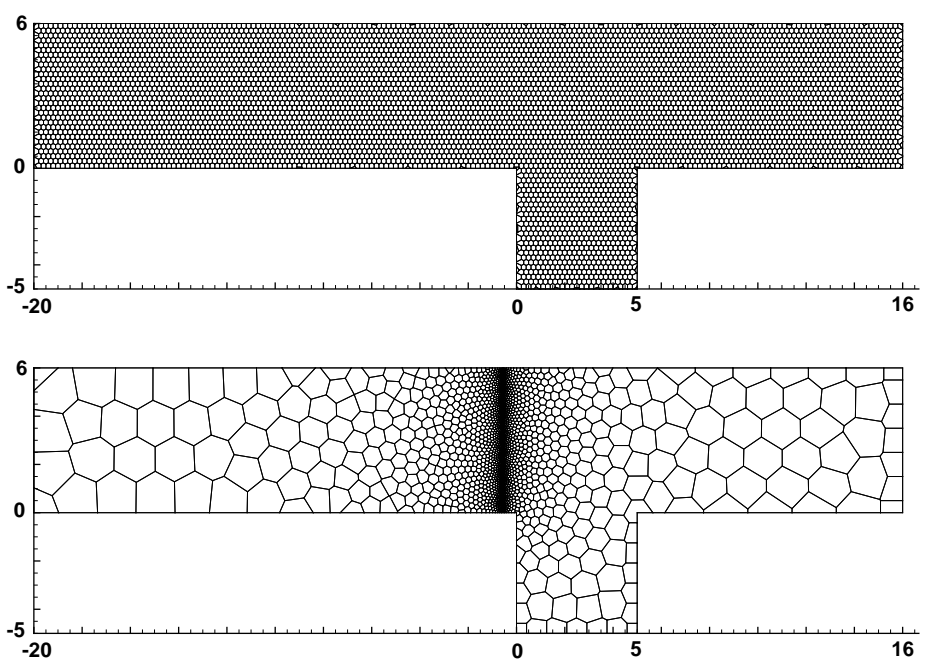

Figure 50: Interaction between a shock and cavity. The initial mesh of the ReALE $N=6051$ (top) and A-ReALE $A_{\text {min }}=2 \times 10^{-3}$ (bottom).

number of mesh cells changes in time to maintain a chosen equi-distribution level. We describe all details related to implementation of our adaptive method.

Using a series of test problems, we have demonstrated superiority of the new adaptive A-ReALE methods in comparison with the standard non-adaptive ReALE method. In our opinion, the main strength of the A-ReALE method is that it combines robustness of ReALE method (due to reconnection) with adaptation capability.

In the future we are planning to explore other monitor functions, for example, the non-dimensional monitor function proposed by Löhner [32]. We also planning to add and remove generators on boundary segments to improve accuracy near the boundary.

\section{Acknowledgments}

This work was performed under the auspices of the National Nuclear Security Administration of the US Department of Energy at Los Alamos National Laboratory under Contract No. DE-AC52-06NA25396. The authors gratefully acknowledge the partial support of the US Department of Energy Office of Science Advanced Scientific Computing Research (ASCR) Program in Applied Mathematics Research and the partial support of the US Department of Energy National Nuclear Security Administration Advanced Simulation and Computing (ASC) Program. LA-UR-15-21737.

We would like to thank R. Loubere, P.-H. Maire, J. Breil, S. Galera, and S. Sambasivan who participated in developing the ReALE methodology as well as 


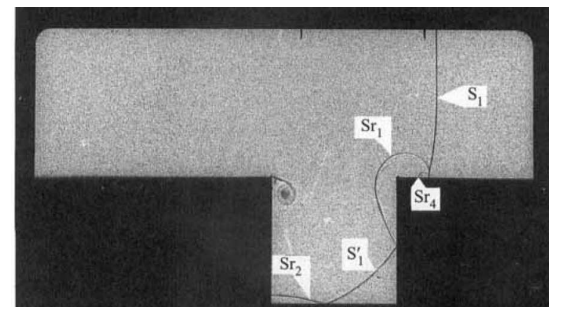

The shadowgraph from the experimental results, [25]
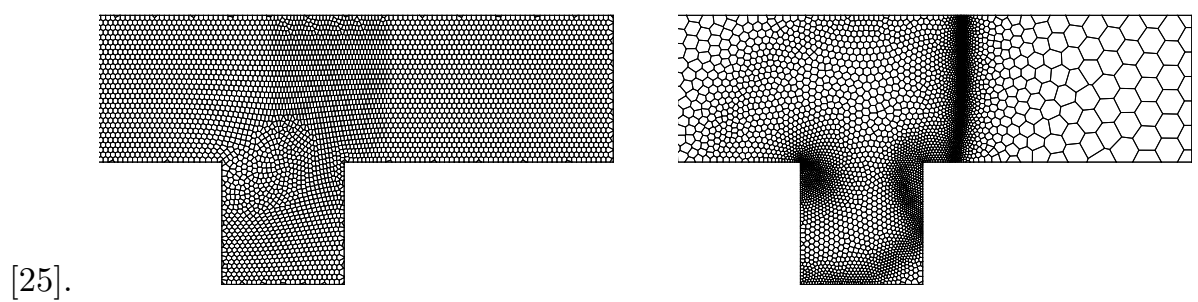

Mesh, ReALE $N=6051$

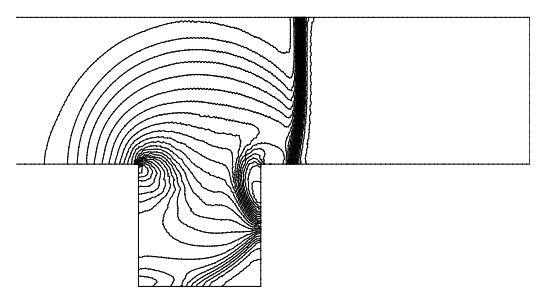

Mesh, A-ReALE $A_{\min }=2 \times 10^{-3}$

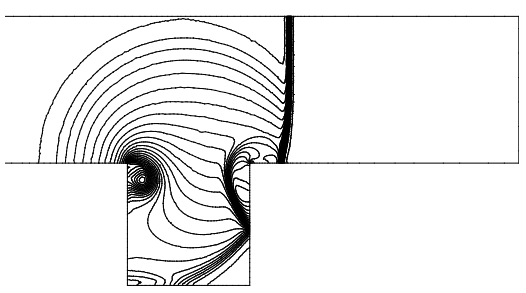

ReALE $N=6051$
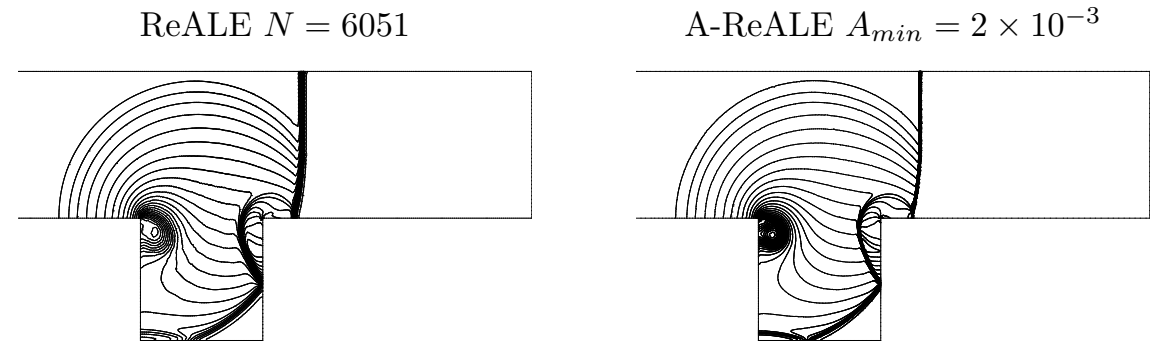

ReALE $N=39593$

A-ReALE $A_{m i n}=1.25 \times 10^{-4}$

Figure 51: Interaction between a shock and cavity. Mesh and pressure contour plots at $t=160$. 


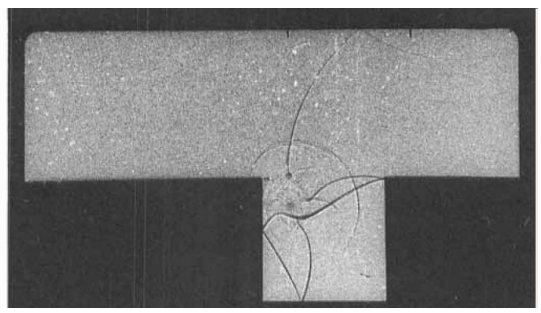

The shadowgraph from the experimental results, [25]
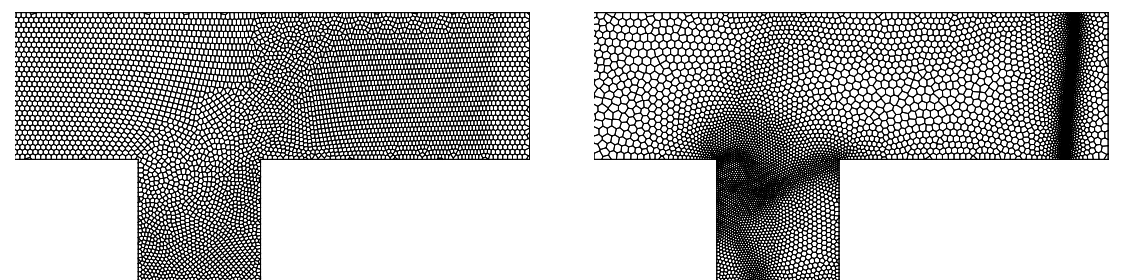

Mesh, ReALE $N=6051$

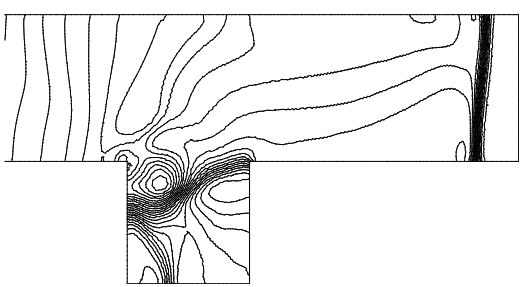

Mesh, A-ReALE $A_{\min }=2 \times 10^{-3}$

ReALE $N=6051$

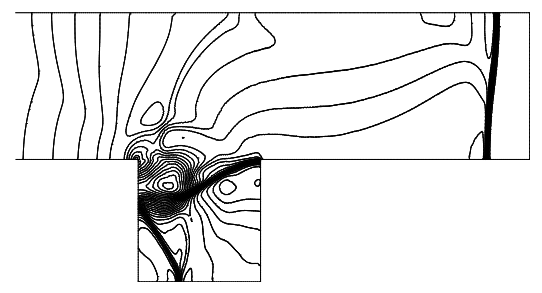

ReALE $N=39593$

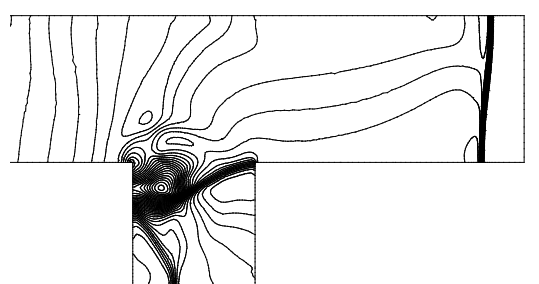

A-ReALE $A_{\text {min }}=2 \times 10^{-3}$

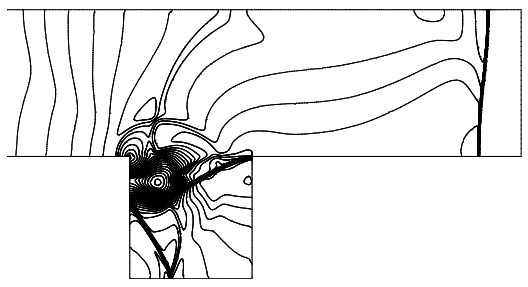

A-ReALE $A_{\min }=1.25 \times 10^{-4}$

Figure 52: Interaction between a shock and cavity. Mesh and pressure contour plots at $t=340$. 


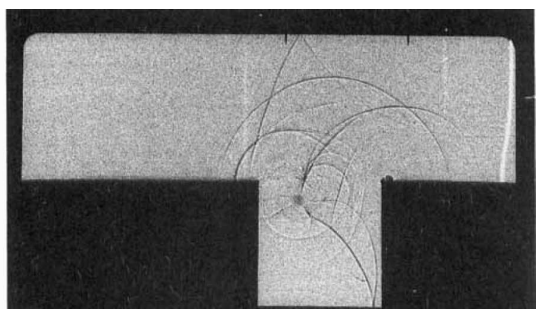

The shadowgraph from the experimental results, [25]
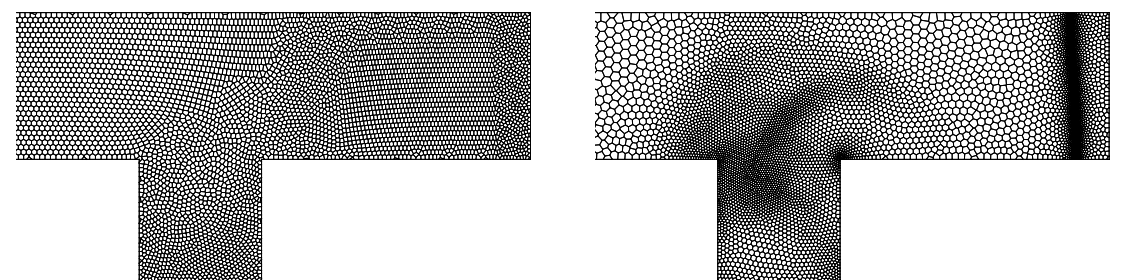

Mesh, ReALE $N=6051$

Mesh, A-ReALE $A_{\min }=2 \times 10^{-3}$
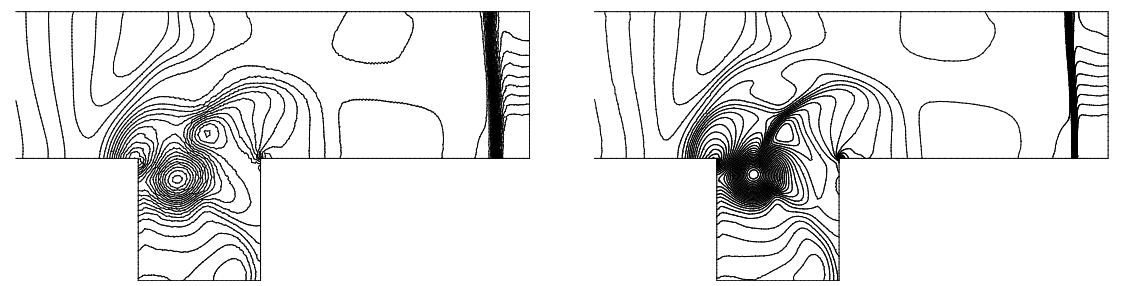

ReALE $N=6051$

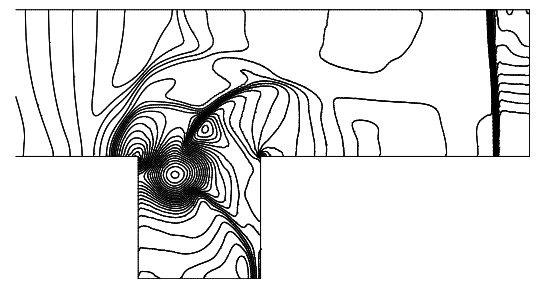

ReALE $N=39593$

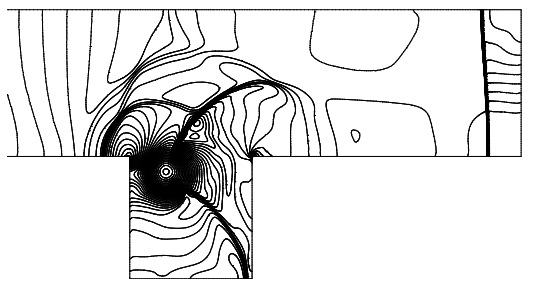

A-ReALE $A_{\min }=1.25 \times 10^{-4}$

Figure 53: Interaction between a shock and cavity. Mesh and pressure contour plots at $t=420$. 
M. Owen, D. Miller, D. Starinshak for numerous discussions related to ReALE. We are also thankful to S. Diot and S. Mao, who participated in initial stages of the developing adaptive ReALE ideas. We thank J. Quirk, S. Li, S. Popinet S. Del Pino and A. Barlow for sharing their thoughts on the efficiency of adaptive methods.

\section{References}

[1] F. Alauzet. High-order methods and mesh adaptation for Euler equations. International journal for numerical methods in fluids, 56(8):1069-1076, 2008.

[2] F. Aurenhammer. Voronoi diagrams - a survey of fundamental geometric data structures. ACM Computing Surveys, 23(3):345-405, 1991.

[3] T. J. Barth. Numerical methods for gasdynamic systems on unstructured meshes. In D. Kroner, M. Ohlberger, and C. Rohde, editors, An introduction to Recent Developments in Theory and Numerics for Conservation Laws, Proceedings of the International School on Theory and Numerics for Conservation Laws, pages 195-284, Berlin, 1997. Lecture Notes in Computational Science and Engineering, Springer.

[4] Anca Belme, Alain Dervieux, and Frédéric Alauzet. Time accurate anisotropic goal-oriented mesh adaptation for unsteady flows. Journal of Computational Physics, 231(19):6323-6348, 2012.

[5] W. Bo and M. Shashkov. R-Adaptive Reconnection-based Arbitrary Lagrangian Eulerian Method - R-ReALE. Technical report, Los Alamos National Laboratory Report LA-UR-15-20094, 2015. Submitted to Journal of Mathematical Study.

[6] J. Breil, T. Harribey, P.-H. Maire, and M. Shashkov. A multi-material ReALE method with MOF interface reconstruction. Computers \& Fluids, 83:115-125, 2013.

[7] Chris J Budd, Weizhang Huang, and Robert D Russell. Adaptivity with moving grids. Acta Numerica, 18:111-241, 2009.

[8] G Carré, S Del Pino, Bruno Després, and Emmanuel Labourasse. A cell-centered Lagrangian hydrodynamics scheme on general unstructured meshes in arbitrary dimension. Journal of Computational Physics, 228(14):5160-5183, 2009.

[9] Siu-Wing Cheng, Tamal Krishna Dey, and Jonathan Richard Shewchuk. Delaunay mesh generation. CRC Press, 2012.

[10] E.A. Dorfi and L.O'C. Drury. Simple adaptive grids for 1-D initial value problems. J. Comput. Phys., 69:175-195, 1987. 
[11] Qiang Du, Vance Faber, and Max Gunzburger. Centroidal Voronoi tessellations: Applications and algorithms. SIAM review, 41(4):637-676, 1999.

[12] Qiang Du, Max Gunzburger, and Lili Ju. Advances in studies and applications of centroidal Voronoi tessellations. Numerical Mathematics: Theory, Methods and Applications, 3(2):119-142, 2010.

[13] Qiang Du and Desheng Wang. Tetrahedral mesh generation and optimization based on centroidal Voronoi tessellations. International journal for numerical methods in engineering, 56(9):1355-1373, 2003.

[14] Qiang Du and Desheng Wang. Recent progress in robust and quality Delaunay mesh generation. Journal of Computational and Applied Mathematics, 195(1):8-23, 2006.

[15] John K Dukowicz and John W Kodis. Accurate conservative remapping (rezoning) for arbitrary Lagrangian-Eulerian computations. SIAM Journal on Scientific and Statistical Computing, 8(3):305-321, 1987.

[16] Pascal Jean Frey and Jean Marechal. Fast adaptive quadtree mesh generation. In 7th International Meshing Roundtable, pages 211-224. Citeseer, 1998.

[17] Allen Gersho. Asymptotically optimal block quantization. Information Theory, IEEE Transactions on, 25(4):373-380, 1979.

[18] J. Grandy. Conservative remapping and region overlays by intersecting arbitrary polyhedra. J. Comput. Phys., 148(2):133-166, 1999.

[19] Peter M Gruber. Optimum quantization and its applications. Advances in Mathematics, 186(2):456-497, 2004.

[20] T. Harribey, J. Breil, P-H. Maire, and M. Shashkov. A sweptintersectionbased remapping method in a ReALE framework. International Journal for Numerical Methods in Fluids, 72:697-708, 2013.

[21] Thibault Harribey, Jérôme Breil, Pierre-Henri Maire, and Mikhail Shashkov. A swept-intersection-based remapping method in a reale framework. International Journal for Numerical Methods in Fluids, 72(6):697708, 2013.

[22] Frédéric Hecht. BAMG: bi-dimensional anisotropic mesh generator. Website: http://www-rocq1. inria. fr/gamma/cdrom/www/bamg/eng. htm, 1998.

[23] C. Hirt, A. Amsden, and J.J.L. Cook. An arbitrary Lagrangian- Eulerian computing method for all flow speeds. Journal of Computational Physics, 14(3):227-253, 1974.

[24] Weizhang Huang and Robert D Russell. Adaptive moving mesh methods. Springer, 2010. 
[25] O. Igra, J. Falcovitz, H. Reichenbach, and W. Heilig. Experimental and numerical study of the interaction between a planar shock wave and a square cavity. Journal of Fluid Mechanics, 313(1):105-130, 1996.

[26] Lili Ju, Qiang Du, and Max Gunzburger. Probabilistic methods for centroidal Voronoi tessellations and their parallel implementations. Parallel Computing, 28(10):1477-1500, 2002.

[27] Lili Ju, Max Gunzburger, and Weidong Zhao. Adaptive finite element methods for elliptic PDEs based on conforming centroidal VoronoiDelaunay triangulations. SIAM Journal on Scientific Computing, 28(6):2023-2053, 2006.

[28] James R Kamm and FX Timmes. On efficient generation of numerically robust Sedov solutions. submitted to Astrophys. J. Suppl. Ser., in review, 2007.

[29] M. Kuchark, M. Shashkov, and B. Wendroff. An efficient linearity-andbound-preserving remapping methods. J. Comput. Phys., 188:462-471, 2003.

[30] K. Lipnikov and M. Shashkov. The error-minimization-based strategy for moving mesh methods. Commun. Comput. Phys., 1(1):53-80, 2006.

[31] Yang Liu, Wenping Wang, Bruno Lévy, Feng Sun, Dong-Ming Yan, Lin Lu, and Chenglei Yang. On centroidal Voronoi tessellation-energy smoothness and fast computation. ACM Transactions on Graphics (ToG), 28(4):101, 2009.

[32] Rainald Löhner. An adaptive finite element scheme for transient problems in cfd. Computer Methods in Applied Mechanics and Engineering, 61(3):323-338, 1987.

[33] Rainald Löhner. Applied CFD Techniques. An Intoroduction based on Finite Element Methods. Wiley, 2001.

[34] R. Loubère, P.-H. Maire, and M. Shashkov. ReALE: A Reconnection Arbitrary-Lagrangian-Eulerian method in cylindrical geometry. Computers \& Fluids, 46(1):59 - 69, 2011.

[35] R. Loubère, P.-H. Maire, M. Shashkov, J. Breil, and S. Galera. ReALE: A Reconnection-based Arbitrary-Lagrangian-Eulerian method. Journal of Computational Physics, 229(12):4724 - 4761, 2010.

[36] James MacQueen. Some methods for classification and analysis of multivariate observations. In Proceedings of the fifth Berkeley symposium on mathematical statistics and probability, volume 1, pages 281-297. California, USA, 1967. 
[37] P.-H. Maire. A high-order cell-centered Lagrangian scheme for twodimensional compressible fluid flows on unstructured meshes. Journal of Computational Physics, 228(7):2391-2425, 2009.

[38] Pierre-Henri Maire, Rémi Abgrall, Jérôme Breil, and Jean Ovadia. A cellcentered Lagrangian scheme for two-dimensional compressible flow problems. SIAM Journal on Scientific Computing, 29(4):1781-1824, 2007.

[39] Hoa Nguyen, John Burkardt, Max Gunzburger, Lili Ju, and Yuki Saka. Constrained CVT meshes and a comparison of triangular mesh generators. Computational geometry, 42(1):1-19, 2009.

[40] Hoa Nguyen, Max Gunzburger, Lili Ju, and John Burkardt. Adaptive anisotropic meshing for steady convection-dominated problems. Computer Methods in Applied Mechanics and Engineering, 198(37):2964-2981, 2009.

[41] A. Okabe, B. Boots, K. Sugihara, and S.N. Nok Chiu. Spatial Tessellations: Concepts and Applications of Voronoi Diagrams. Wiley Series in Probability and Statistics, 2000.

[42] Stéphane Del Pino. Metric-based mesh adaptation for 2D Lagrangian compressible flows. Journal of Computational Physics, 230(5):1793-1821, 2011.

[43] Jonathan Quinn, Feng Sun, Frank C Langbein, Yu-Kun Lai, Wenping Wang, and Ralph R Martin. Improved initialisation for centroidal Voronoi tessellation and optimal Delaunay triangulation. Computer-Aided Design, 44(11):1062-1071, 2012.

[44] M.Yu. Shashkov and A.V. Solovjov. A generalization of the notion of Dirichlet cell for non-convex domain. Technical report, Keldysh Institute of Applied Mathematics, USSR Academy of Sciences, Moscow, Russia, Preprint No. 32, 1990 (in Russian), 1990.

[45] M.Yu. Shashkov and A.V. Solovjov. Numerical simulation of twodimensional flows by the free-lagrangian flow simulations. Technical report, Mathematisches Institut, Technische Universitt, Munchen, 1991, Report TUM-M9105. Available at ¡http://cnls.lanl.govshashkovi, 1991.

[46] G.A. Sod. Numerical Methods in Fluid Dynamics. Initial and initial boundary-value problems. Cambridge University Press, 1985.

[47] D.P. Starinshak, J.M. Owen, and J.N. Johnson. A new parallel algorithm for constructing Voronoi tessellations from distributed input data. Computer Physics Communications, 185:3204-3214, 2014.

[48] H. Tang and T. Tang. Adaptive mesh methods for one-and two-dimensional hyperbolic conservation laws. SIAM Journal on Numerical Analysis, 41(2):487-515, 2003. 
[49] Hua-Zhong Tang. A moving mesh method for the euler flow calculations using a directional monitor function. Commun. Comput. Phys, 1:656-676, 2006.

[50] Jane Tournois, Pierre Alliez, and Olivier Devillers. 2D centroidal Voronoi tessellations with constraints. Numerical Mathematics: Theory, Methods E Applications, 3(2), 2010.

[51] François Vilar. A high-order Discontinuous Galerkin discretization for solving two-dimensional Lagrangian hydrodynamics. Theses, Université Sciences et Technologies - Bordeaux I, November 2012. https://tel.archivesouvertes.fr/tel-00765575. 
LaTeX Source Files
Click here to download LaTeX Source Files: shashkov_JCOMP-D-15-00326-R1.tar.gz

LaTeX Source Files
Click here to download LaTeX Source Files: shashkov_JCOMP-D-15-00326-R1.tar.gz z . .

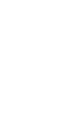
.

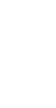

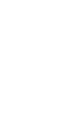
.

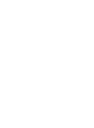

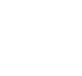

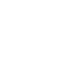

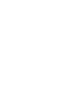

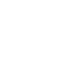

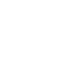

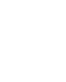

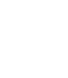

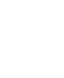

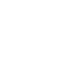

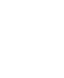

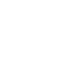

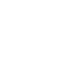

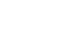

